\title{
Smart Switching Bilateral Filter with Estimated Noise Characterization for Mixed Noise Removal
}

\author{
Kriengkri Langampol, ${ }^{1}$ Kanabadee Srisomboon, ${ }^{1}$ \\ Vorapoj Patanavijit, ${ }^{2}$ and Wilaiporn Lee $\mathbb{i D}^{1}$ \\ ${ }^{1}$ Communication and Computer Network Research Group (C2NRG), Electrical Engineering, Department of Electrical and \\ Computer Engineering, Faculty of Engineering, King Mongkut's University of Technology North Bangkok, Thailand \\ ${ }^{2}$ Department of Electrical and Electronic Engineering, Faculty of Engineering, Assumption University, Bangkok, Thailand
}

Correspondence should be addressed to Wilaiporn Lee; wilaiporn.l@eng.kmutnb.ac.th

Received 26 February 2019; Accepted 8 May 2019; Published 28 May 2019

Academic Editor: Erik Cuevas

Copyright (C) 2019 Kriengkri Langampol et al. This is an open access article distributed under the Creative Commons Attribution License, which permits unrestricted use, distribution, and reproduction in any medium, provided the original work is properly cited.

\begin{abstract}
Traditionally, several existing filters are proposed for removing a specific type of noise. However, in practice, the image communicated through the communication channel may be contaminated with more than one type of noise. Switching bilateral filter $(\mathrm{SBF})$ is proposed for removing mixed noise by detecting a contaminated noise at the concerned pixel and recalculates the filter parameters. Although the filter parameters of SBF are sensitive to type and strength of noise, the traditional SBF filter has not taken the strength into account. Therefore, the traditional SBF filter cannot remove the mixed noise efficiently. In this paper, we propose a smart switching bilateral filter (SSBF) to outperform a demerit of traditional SBF filter. In the first stage of SSBF, we propose a new scheme of noise estimation using domain weight (DW) pattern which characterizes the distribution of the different intensity between a considered pixel and its neighbors. By using this estimation, the types of mixed noises and their strength are estimated accurately. The filter parameters of SBF are selected from the table where the spatial weight and radiometric weight are already learned. As a result, SSBF can improve the performance of traditional SBF and can remove mixed noises efficiently without knowing the exact type of contaminated mixed noise. Moreover, the performance of SSBF is compared to the optimal SBF filter (OSBF) where OSBF sets the optimal value of filter parameters on the contaminated mixed noise and three new filters - blockmatching and 3D filtering (BM3D), nonlocal sparse representation (NCSR), and trilateral filter (TF). The simulation results showed that the performance of SSBF outperforms BM3D, NCSR, TF, and SBF and is near to optimal SBF filter, even if the SSBF does not know the type of mixed noise.
\end{abstract}

\section{Introduction}

Currently, a high quality image is used in several applications such as security system or medical diagnosis. The quality of image affects these applications directly; e.g., the intruders cannot be identified if the quality of image is reduced. Moreover, this image can be communicated through communication technology where the transmitted image may be contaminated by noises. Therefore, the performance of the received image degrades. Filtering is an important function of image processing which is used to remove noises from the image [1-3]. Generally, the filter is designed for a specific type of noise. Then, to restore the desired image from the received image, the filtering technique should be selected on the type of detected noise.

The types of noise that contaminate the image can be classified into two groups including linear noise and nonlinear noise [2-4]. Gaussian noise is the well-known types of linear noise whereas speckle noise, salt-and-pepper noise, and uniform impulse noise are nonlinear noise. Gaussian noise can be characterized by its distribution with mean and variance values. In a nonlinear group, salt-and-pepper noise corrupts the image's pixels randomly and sparsely which makes some pixel change to bright or dark. Uniform impulse noise is considered by replacing a portion of some image pixel values with random values. Speckle noise is characterized by 
signal-dependent noise where the noise corrupts the image in the form of multiplicative noise [5-12].

As mentioned earlier, most of the denoising techniques can be used to perform noise reduction when the image is contaminated by a single type of noise [4]. Nevertheless, in practice, the images may be contaminated by more than one type of noise at the same time. Then, former image noise removal techniques cannot remove mixed noises efficiently. Recently, there are a few numbers of filters that are proposed to remove a mixed noise. Therefore, in this work, the mixed noise removal technique was focused.

To remove mixed noise, at least two filters are implemented for eliminating a specific type of noise. Therefore, the type of corrupted noise at the considered pixel should be identified accurately in order to select the appropriate filter. The noise removal technique can be categorized into two groups - the spatial domain and transform domain. In the spatial domain, the image is performed noise removal without transforming the information to other domains while noise removing based domain transformation needs to convert image to other domains and then perform noise removal.

In the spatial domain based technique, several types of filter are proposed to remove mixed noise. In $[6,13]$, the fuzzy rule is utilized to formulate weight averaging where the weight of noisiness and weight of similarity between the considered pixel and neighbors were taken into account. Nonlocal mean (NLM) approach [14] is exploited. By utilizing NLR; not only are neighbors considered, but patches which contain similar details are also determined. However, only using NLM cannot achieve high performance on local patches grouping under high strength of the noise. On the other hand, noise removal techniques based on the bilateral filter (BF) [15-17] - trilateral filter (TF) [18-21] and switching bilateral filter (SBF) [22-25] —are proposed. These techniques are proposed to improve the performance of $\mathrm{BF}$ under impulsive noise. It should be noted that BF works well on Gaussian noise. Based on the hybrid scheme of TF, a new parameter-impulsive weighting function-is taken into the account where this parameter is used to perform a noise removal on the pixel contaminated by impulsive noise. However, TF filter cannot effectively distinguish the noise type efficiently when the strength of noise is high. Moreover, TF does not consider other types of noise, e.g., salt-and-pepper and speckle noise. Therefore, when these types of noise corrupt the image, TF removes the noise ineffectively. On the other hand, SBF is proposed to remove two types of noise including Gaussian noise and salt-andpepper noise. SBF determines the type of contaminated noise using the sorted quadrant median vector (SQMV) technique and reference median (RM) technique [22]. Then, it switches the predetermined filter parameters-spatial weight $\left(\sigma_{\mathrm{S}}\right)$ and radiometric weight $\left(\sigma_{\mathrm{R}}\right)$-on the detected type of noise [25]. In our previous work [25], we evaluated the performance of SBF filter for several types of images, noise types, and noise strength. Although switching bilateral filter (SBF) has merit to remove both linear and nonlinear noise, its demerit is the sensitivity of $\sigma_{\mathrm{S}}$ and $\sigma_{\mathrm{R}}$ which cannot be fixed and needs to be re-calculated on the detected type and level of noise.
Sparse representation [26] is one of the most studied methods for image denoising scheme. By transforming the image into other domains, true signal and noise can be distinguished accurately. Based on the advantages of sparse representation and NLM, several filters [9, 27-32], such as weighted encoding with sparse block-matching and 3D filtering (BM3D) [28], nonlocal sparse representation (NCSR) [29], nonlocal regularization (WESNR) [9] and fuzzy impulsive additive noise suppression using sparse representation (F_IANS_SR) [30], combine these methods in order to achieve high performance of mixed noise removal. The drawbacks of filters based on sparse representation and NLM combination are described in Section 2. Among these techniques, BM3D and NCSR are used to compare to our proposed technique since they show high noise removal performance and are used in several kinds of literature in performance comparison.

In this paper, an alternative SBF filter, smart switching bilateral filter (SSBF), and an estimated noise characterization scheme for noise estimation were proposed. It should be noted that once the noise type and strength are efficiently estimated, the SSBF filter removes the mixed noise with the appropriate $\sigma_{\mathrm{S}}$ and $\sigma_{\mathrm{R}}$. The proposed SSBF filter improves the performance of SBF filter by learning the pattern of mixed noise and varying the value of $\sigma_{\mathrm{S}}$ and $\sigma_{\mathrm{R}}$ on the detected mixed noise. Therefore, the contribution of this paper is twofold. First, we propose an estimated noise characterization scheme to estimate the type and strength of mixed noise. To determine the noise type and strength, joint impulsivity function on two-dimensional data is implemented to generate the distribution pattern of mixed noise, domain weight (DW) pattern. The DW pattern characterizes the distribution of the difference between the intensity of center pixel and neighbors. Instead of estimating the type of mixed noise from the intensity of image pixels, the estimated noise characterization scheme gives an accurate estimation since each mixed noise type has a specific distribution pattern of DW. Second, we address the issues of traditional SBF filter by generating the table of $\sigma_{\mathrm{S}}$ and $\sigma_{\mathrm{R}}$ for several types and strengths of mixed noises. After the type and strength of mixed noise are estimated, the appropriate $\sigma_{\mathrm{S}}$ and $\sigma_{\mathrm{R}}$ are selected from the table to perform a noise removal. To evaluate the performance of SSBF, we simulate the SBF filter which sets the optimal value on the contaminated mixed noise and called optimal SBF filter (OSBF). Moreover, three new filters-block-matching and 3D filtering (BM3D), nonlocal sparse representation (NCSR), and trilateral filter (TF) - are also compared. As a result, SSBF gives a high performance of mixed noise removal and nearly to the OSBF filter without any knowledge about the contaminated mixed noise. It should be mentioned that the OSBF filter needs to know the exact noise type and strength to calculate $\sigma_{\mathrm{S}}$ and $\sigma_{\mathrm{R}}$ to perform filtering.

The performances of SSBF under mixed noise environments are considered. Four cases of mixed noise include case A (Gaussian and salt-and-pepper), case B (Gaussian and uniform impulse), case $\mathrm{C}$ (speckle and salt-and-pepper), and case D (speckle and uniform impulse). We will consider the sixteen levels of noise strength. In the simulation, we consider 
our technique performance pass through peak signal-tonoise ratio (PSNR), the structural similarity index (SSIM), and mean-squared error (MSE) [33]. Simulation results show that the SSBF gives the best PSNR for all noise cases.

This paper is organized as follows. Section 2 presents switching the bilateral filter. Section 3 presents a smart switching bilateral filter with estimated noise characterization. Section 4 presents the learning process, noise estimation evaluation, and the simulations results. Finally, a brief conclusion is given in Section 6.

\section{Related Works}

The efficient method for removing mixed noise is to exploit several filters which are appropriate to the considered noises. In general, at least two filters are applied to remove mixed noise. Applying the appropriate filter to the detected noise, noise type at the considered pixel needs to be identified for selecting the appropriate filter. Therefore, the noise estimation plays an important role in mixed noise removal scheme. Traditionally, the noise removal technique can be categorized into two groups-spatial domain and transform domain.

By removing noise on the spatial domain, the filters remove the noise from the image directly. In $[6,13]$, mixed noise removal techniques based on the fuzzy rule are proposed. To remove mixed noise, these filters formulate weight averaging by utilizing a weight of similarity between considered pixel and neighbors and weight of noisiness. Although these filters can remove mixed noise while preserving edge and detail of the image, they cannot achieve a high noise removal performance since only local neighbor pixels cannot give appropriate weight under high strength of noise. Moreover, by exploiting fuzzy theorem, several parameters need to be taken into the account, which comes at a cost of the computational burden.

Since only local information of image pixels cannot achieve high performance, a nonlocal mean (NLM) filter [14] is proposed to address this issue. By utilizing a concept of nonlocal method, similar image patches are taken into account in order to perform noise removing using weight average algorithm. However, NLM filter also suffers from high strength of noise since the local patches cannot be grouped accurately.

On the other hand, bilateral filter [15-17] also has been extensively studied and modified for mixed noise removal. Trilateral filter (TF) [18-21], switching bilateral filter (SBF) [22-25], and adaptive bilateral filter (ABF) [34] are the modification of traditional bilateral filter. TF filter performs a noise removal based on a hybrid scheme where traditional BF and Rank-Ordered Absolute Differences (ROAD) statistic are exploited. From our study, we found that TF filter gives a poor performance under high strength of mixed noise between Gaussian noise and impulsive noise because the type of noise at the considered pixel cannot be identified effectively. Moreover, when the type of contaminated noise is not Gaussian or impulsive noise, e.g., salt-and-pepper and speckle noise, the TF filter cannot remove noise efficiently. SBF is an improved method of a bilateral tradition filter which has a demerit in removing nonlinear noise. Different from TF, SBF determines the type of contaminated noise using the sorted quadrant median vector (SQMV) technique and reference median (RM) technique [22]. However, the performance of SBF filter is sensitive to the strength of the noise. Once the strength of noise changes, SBF filter cannot maintain its performance at a high level. ABF performs a mixed noise removal by exploiting bilateral filter with adaptive scheme and impulse noise detector. The parameter of BF filters is adapted utilizing artificial bee colony algorithm. Although ABF works well under high strength of noise, it cannot preserve the detail of image which contains smooth regions, e.g., Lena and Pepper.

Based on domain transformation, sparse representation can be used to express the behavior of noise. By transforming the domain, the desired signal gives a high magnitude of transform coefficient while the noise gives low magnitude. In [26], image denoising based on wavelet is proposed by applying wavelet transform to local neighborhoods specified by similarity coefficient. Then, each sub-band of wavelet is performed noise removal through adapting scheme.

In [28], image denoising technique based on sparse representation with extensive use of a nonlocal method-blockmatching and 3D filtering (BM3D) - is proposed. A sparse transformation is applied on similar $2 \mathrm{D}$ image patches based on the nonlocal method. These patches are formed as a $3 \mathrm{D}$ array which is applied to a collaborative filtering process. Then, the output from the collaborative filtering process is performed an inverse 3D transformation to reconstruct the image into the traditional domain. However, BM3D gives a poor performance under high strength of noise caused by an inaccurate block-matching. In [27], the concept of $\mathrm{BM} 3 \mathrm{D}$ is modified to perform noise removal on video data where the technique is known as "BM4D." Instead of creating $3 \mathrm{D}$ arrays formed by similar image patches, BM4D uses 3D information of video, i.e., sequence patches, to create 4D data structure. Then, the collaborative filter is applied to $4 \mathrm{D}$ data to remove noise. It can be noticed that a matching process is an important task of BM3D and BM4D. If the block-matching process is inaccurate, $\mathrm{BM} 3 \mathrm{D}$ and $\mathrm{BM} 4 \mathrm{D}$ cannot achieve high performance.

In [30-32], the performance of traditional BM3D is improved by applying the bilateral filter to reduce artifacts created by impulse noise and Gaussian noise suppressing process. However, their performance still suffers from bilateral filter's disadvantages where its performance is sensitive to the strength of noise. It can be noticed that the mixed noise removal technique based on sparse representation cannot make an accurate distinguishing between true signal and noise under high strength of the noise.

In [9], the mixed noise removal technique based on the encoding scheme is proposed. Different from others, impulse noise and Gaussian noise are removed simultaneously through encoding scheme where noisy patch is encoded over the prelearned dictionary. Although it gives high performance on normal images, it fails to maintain high performance on high details image. In [29], the nonlocal method is combined with sparse coding scheme to address a lacking of smoothness issue of traditional nonlocal method. The difference between noisy image and the unknown desired 


$$
\begin{aligned}
& \text { if }\left(\left|x_{i, j}-R M\right| \geq \mathrm{Tk} 1\right) \\
& S_{1}=1, S_{2}=1 ; \quad \quad / / \text { nonlinear noise } \\
& \text { else if }\left(\left|x_{i, j}-R M\right| \geq \mathrm{Tk} 2\right) \\
& S_{1}=1, S_{2}=0 ; \quad \quad / / \text { linear noise } \\
& \text { else } \\
& S_{1}=0, S_{2}=0, y_{i, j}=x_{i, j} \quad / / \text { noise free }
\end{aligned}
$$

Algorithm 1: Noise estimation of SBF filter.

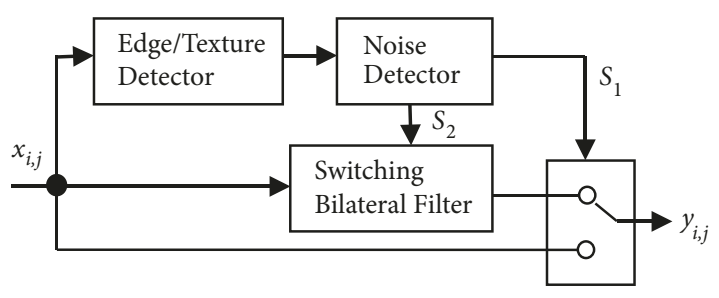

FIGURE 1: Switching bilateral filter (SBF) block diagram.

image is evaluated utilizing sparse coding. This filter is known as nonlocal sparse representation (NCSR). However, artifacts still occur.

\section{Switching Bilateral Filter}

A switching bilateral filter (SBF) [22] is an improved method of a tradition bilateral filter which has a demerit in removing nonlinear noise. As shown in Figure 1, the framework of SBF filter can be separated into two stages including detecting and filtering. First, the detected image $\left(x_{i, j}\right)$ is separated into several $(2 N+1) \times(2 N+1)$ windows $(W)$ as shown in Figure $2(a)$. These windows identified whether the information is edge of image or texture. After the type of information is detected, the contaminated noise in the window is determined by noise detector using sorted quadrant median vector (SQMV) [22].

To determine $S Q M V$, the window $(W)$ is separated into four $(N+1) \times(N+1)$ subwindows $\left(W_{k}\right)$ and calculates the median $\left(m_{k}\right)$ of each subwindow as shown in Figure 2(b). Then, these median values are used to calculate a reference median $(R M)$ which will be used to identify whether the considered window is contaminated with noise or is noise free. Since the type of noise is detected, then the appropriate values of SBF parameters can be determined.

Therefore, $m_{k}$ of subwindows $\left(W_{k}\right)$ can be expressed as

$$
m_{k}=\operatorname{median}\left\{W_{k}\right\}, \quad k=1 \text { to } 4 .
$$

Then, $S Q M V$ is given by

$$
S Q M V=\left[m_{1}, m_{2}, m_{3}, m_{4}\right] .
$$

The reference median $(R M)$ is given as

$$
\begin{aligned}
& R M \\
& = \begin{cases}\frac{m_{3}+m_{2}}{2}, & S Q M C \leq \mathrm{T}_{\mathrm{RM}} \\
m_{3}, & S Q M C \geq \mathrm{T}_{\mathrm{RM}}, A V G \in\left(m_{3}, m_{4}\right) \\
m_{2}, & S Q M C \geq \mathrm{T}_{\mathrm{RM}}, A V G \in\left(m_{1}, m_{2}\right)\end{cases}
\end{aligned}
$$

where

$$
\begin{aligned}
S Q M C & =m_{3}-m_{2} \\
A V G & =\frac{m_{1}+m_{2}+m_{3}+m_{4}}{4} .
\end{aligned}
$$

To determine the type of noise, the absolute difference between a determined pixel and $R M$ is compared to the thresholds (Tk1 and Tk2) as shown in Algorithm 1. Tk1=30 and Tk2=15 are thresholds for identifying nonlinear noise and linear noise. As described in [22], the appropriated threshold of Tk1 and Tk2 is within the range of $[25,30]$ and $[5,10,15]$, respectively. Therefore, in this work, the values Tk1 and Tk2 are obtained from the experimental study using 10 images. The optimal values of Tk1 and Tk2, which distinguish the nonlinear noise effectively, of those images are averaged. Then, the value of Tk1 and Tk2 is set as 30 and 15, respectively.

Second, the parameters from the first stage are then applied to the filter to remove noise from the image's window. The output of $\operatorname{SBF}\left(y_{i, j}\right)$ can be calculated by

$$
\begin{aligned}
& y_{i, j} \\
& = \begin{cases}\frac{\sum_{s=-N}^{N} \sum_{t=-N}^{N} F_{G}(s, t) F_{S R}(s, t) x_{i+s, j+t}}{\sum_{s=-N}^{N} \sum_{t=-N}^{N} F_{G}(s, t) F_{S R}(s, t)} & \text { noisy } \\
x_{i, j}, & \text { free noise }\end{cases} \\
& F_{G}(s, t)=e^{-\left(\left((i-s)^{2}+(j-t)^{2}\right) / 2 \sigma_{S}^{2}\right)} \\
& F_{S R}(s, t)=e^{-\left(\left(I-x_{i+s, j+t}\right)^{2} / 2 \sigma_{R}^{2}\right)}
\end{aligned}
$$

where $N$ is the window size, $x$ is input pixel, $I=x_{i, j}$ for linear noise $\left(\mathrm{S}_{1}=1\right.$ and $\left.\mathrm{S}_{2}=0\right), I=\mathrm{R} M$ for nonlinear noise $\left(\mathrm{S}_{1}=1\right.$ and $\left.\mathrm{S}_{2}=1\right), y_{i, j}=x_{i, j}$ for noise free $\left(\mathrm{S}_{1}=\right.$ $0), s, t=-N$ to $N, \sigma_{\mathrm{R}}$ and $\sigma_{\mathrm{S}}$ are radiometric weight and spatial weight, respectively.

For tradition SBF filter, radiometric weight $\left(\sigma_{\mathrm{R}}\right)$ and spatial weight $\left(\sigma_{\mathrm{S}}\right)$ are the important parameters and the 


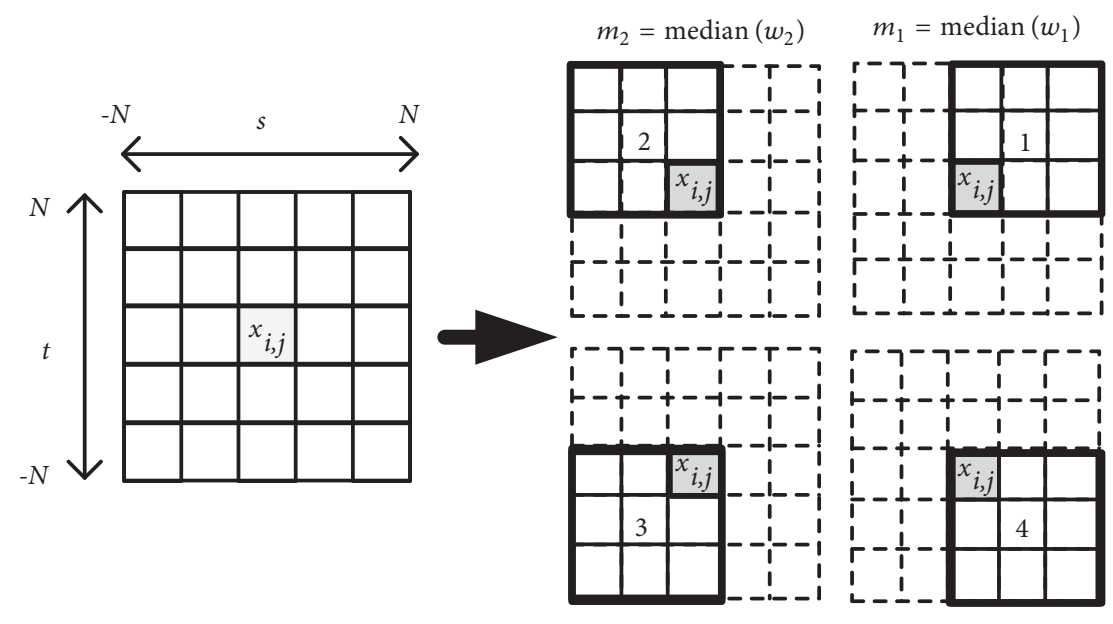

(a)

(b)

FIGURE 2: Example of $S Q M V$ calculation: (a) the $W$ window; (b) four subwindows.

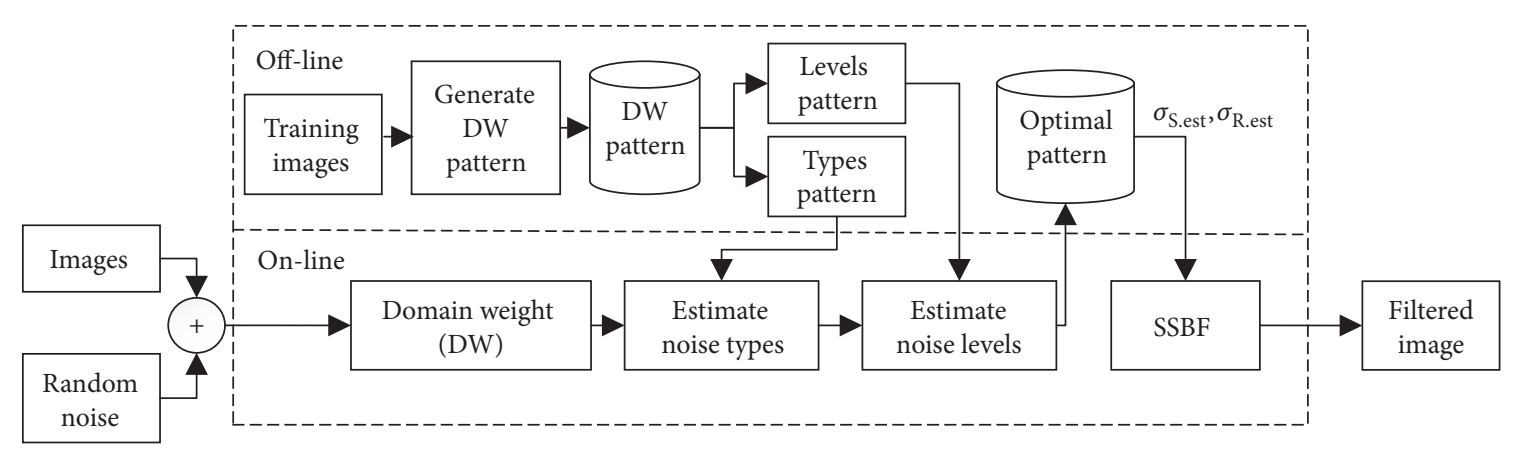

FIGURE 3: Block diagram of smart switching bilateral filter with estimated noise characterization scheme.

values of these parameters affect the performance of the filter. Inside the SBF filter, there are range and domain filter which perform a noise removal. By using the result from noise identification, the range filter recalculates the filter weight for the detected noise-linear and nonlinear noise. In our study [25], the radiometric weight $\left(\sigma_{\mathrm{R}}\right)$ and spatial weight $\left(\sigma_{\mathrm{S}}\right)$ need to be varied on the type of image, the type of noise, and the strength of noise. If the values of radiometric weight $\left(\sigma_{\mathrm{R}}\right)$ and spatial weight $\left(\sigma_{\mathrm{S}}\right)$ are not appropriate to the mentioned factors, the performance of SBF filter is poor. It can be mentioned that the performance of SBF is influenced by the noise identification and the filter weights. If noise is identified accurately, the filter performs noise removal with the appropriate weights. On the other hand, if noise is identified inaccurately, the SBF filter gives poor noise removal performance. Moreover, the filter weights are also fixed; therefore, the SBF filter cannot maintain its performance when the strength of noise changes.

In practice, the image may be contaminated by different types of noise at the same time, which is referred to as mixed noise. However, existing filters are proposed to remove the specific type of noise; e.g., the median filter is used to remove only a nonlinear noise. If the image is contaminated by mixed noise-linear noise and nonlinear noise-the median filter gives poor performance $[35,36]$.

\section{Smart Switching Bilateral Filter with Estimated Noise Characterization}

In this section, the detail of smart switching bilateral filter (SSBF) is described. Since the performance of SBF filter relates on the spatial weight $\left(\sigma_{S}\right)$ and radiometric weight $\left(\sigma_{\mathrm{R}}\right)$ which need to be varied on the type and strength of noises, then, in this paper, we propose a new scheme of mixed noise estimation by implementing joint impulsivity function on two types of dimensional data to generate the pattern and domain weight (DW) which is used to characterize the type of mixed noise. By characterizing the distribution of the difference between center pixel and neighbors of DW, the type and strength of mixed noise can be estimated efficiently.

The framework of the proposed filter can be separated into two modes-offline and online mode, as shown in Figure 3. In an offline mode, the optimal values of SBF filter-radiometric weight $\left(\sigma_{\mathrm{R}}\right)$ and spatial weight $\left(\sigma_{\mathrm{S}}\right)$-and DW pattern for learned images are generated. In our simulation, the DW patterns are learned from 4 standard types of 
images under 16 different strengths of noises where the detail will be described later. In an online mode, firstly, we determined whether detected image is contaminated by mixed noise using the proposed estimated noise characterization scheme. Once a mixed noise is detected, the type and strength are estimated for selecting the appropriate $\sigma_{\text {R.est }}$ and $\sigma_{\text {S.est }}$ to perform a mixed noise removal.

4.1. Offline Mode. In this subsection, we describe the framework of the offline mode including a generation of DW patterns for mixed noise image and optimal values-radiometric weight $\left(\sigma_{\text {R.est }}\right)$ and spatial weight $\left(\sigma_{\text {S.est }}\right)$-for images in the knowledge base. The DW patterns are generated by determining the difference between the center pixel and neighbors using the theoretical of rank-ordered relative differences (ROAD) [21] given by

$$
\operatorname{ROAD}(m)=\sum_{i=1}^{4} r_{i}(m)
$$

where $r_{i}(m)$ is $i^{\text {th }}$ smallest $d_{(i, j)}$ for $j \in \Omega_{i}(N), N$ is windows size, and $d_{(i, j)}=|y(i)-y(j)|$.

Then, we propose a new parameter-domain weight (DW) - to estimate mixed noise type and strength by implementing joint impulsivity function on two types of dimensional data. Therefore, the DW can be expressed as

$$
\begin{aligned}
& D W\left(x_{i, j}, y_{i . j}\right) \\
& =\sum_{i=-N}^{N} \sum_{j=-N}^{N}\left[1-e^{-((R O A D(x)+R O A D(y)) / 2)^{2} / 2 \sigma_{j}^{2}}\right] \\
& D W=\text { Ceiling }\left(D W_{(x, y)}\right)
\end{aligned}
$$

where $x_{i, j}$ is the magnitude of center pixel, $y_{i, j}$ is neighborhood magnitude, and $\sigma_{j}$ is the joint variance of the signal element at position $x$ under consideration.

As depicted in the equation, the DW value shows the difference between the center pixel and neighbor pixels. For mixed noise of gray-scale image, the value of DW is between 0 and 25. The value of DW can be used to determine the type and strength of noise. When the magnitude of considered pixel is equal to the neighbor pixels, DW is 0 . On the other hand, if the magnitude of considered pixel is absolutely different from the neighbor pixels-equal to $255-\mathrm{DW}$ is 25 . After the DW values are calculated from all windows of the detected image, the distribution pattern of DW values-DW pattern-is generated by categorizing into 25 groups, which is shown in Table 1, for determining the type and strength of mixed noise. Each type of noise has a particular DW pattern. Although it combines with other types of noise, the DW pattern can be used to identify the type of mixed noise if it is already learned.

As mentioned earlier, radiometric weight $\left(\sigma_{\mathrm{R}}\right)$ and spatial weight $\left(\sigma_{\mathrm{S}}\right)$ are the key factors of SBF filter. After the type of noise is identified, e.g., when the image is corrupted by a single noise, the values of $\sigma_{\mathrm{R}}$ and $\sigma_{\mathrm{S}}$ should be set

\begin{tabular}{|c|c|}
\hline No. & Range of DW \\
\hline 1 & $0.00-1.00$ \\
\hline 2 & $1.01-2.00$ \\
\hline 3 & $2.01-3.00$ \\
\hline 4 & $3.01-4.00$ \\
\hline 5 & $4.01-5.00$ \\
\hline 6 & $5.01-6.00$ \\
\hline 7 & $6.01-7.00$ \\
\hline 8 & $7.01-8.00$ \\
\hline 9 & $8.01-9.00$ \\
\hline 10 & $9.01-10.00$ \\
\hline 11 & $10.01-11.00$ \\
\hline 12 & $11.01-12.00$ \\
\hline 13 & $12.01-13.00$ \\
\hline 14 & $13.01-14.00$ \\
\hline 15 & $14.01-15.00$ \\
\hline 16 & $15.01-16.00$ \\
\hline 17 & $16.01-17.00$ \\
\hline 18 & $17.01-18.00$ \\
\hline 19 & $18.01-19.00$ \\
\hline 20 & $19.01-20.00$ \\
\hline 21 & $20.01-21.00$ \\
\hline 22 & $21.01-22.00$ \\
\hline 23 & $22.01-23.00$ \\
\hline 24 & $23.01-24.00$ \\
\hline 25 & $24.01-25.00$ \\
\hline
\end{tabular}
appropriately in order to achieve the highest performance. Therefore, in our simulation, we learn the DW pattern for
TABLE 1: Range of DW value for each group.

4 types of mixed noise under 16 levels of noise strength. After the DW patterns are generated, then we determine the appropriate values of radiometric weight $\left(\sigma_{\text {R.est }}\right)$ and spatial weight $\left(\sigma_{\text {S.est }}\right)$ for each DW pattern.

4.2. Online Mode. In an online mode, as shown in Figure 3, the detected mixed noisy image is first determined by using the proposed estimated noise characterization scheme. The DW pattern of detected image is generated from calculated DW values. Then, it is compared to learned DW patterns in the knowledge base to identify the type of mixed noise. After the type of mixed noise is determined, the DW pattern of received image is compared with DW patterns in the considered group of mixed noise to determine the strength. Therefore, the estimated level of mixed noise strength is used to select the value of estimate radiometric weight $\left(\sigma_{\text {R.est }}\right)$ and spatial weight $\left(\sigma_{\text {S.est }}\right)$.

Therefore, the outputs from the estimation section are $\sigma_{\text {R.est }}$ and $\sigma_{\text {S.est }}$ which are used by the SBF filter to perform mixed noise removal. The output of $\operatorname{SBF}\left(y_{i, j}\right)$ can be calculated by

$$
\begin{aligned}
& y_{i, j} \\
& = \begin{cases}\frac{\sum_{s=-N}^{N} \sum_{t=-N}^{N} F_{G}(s, t) F_{S R}(s, t) x_{i+s, j+t}}{\sum_{s=-N}^{N} \sum_{t=-N}^{N} F_{G}(s, t) F_{S R}(s, t)}, & \text { noisy } \\
x_{i, j}, & \text { free noise }\end{cases}
\end{aligned}
$$




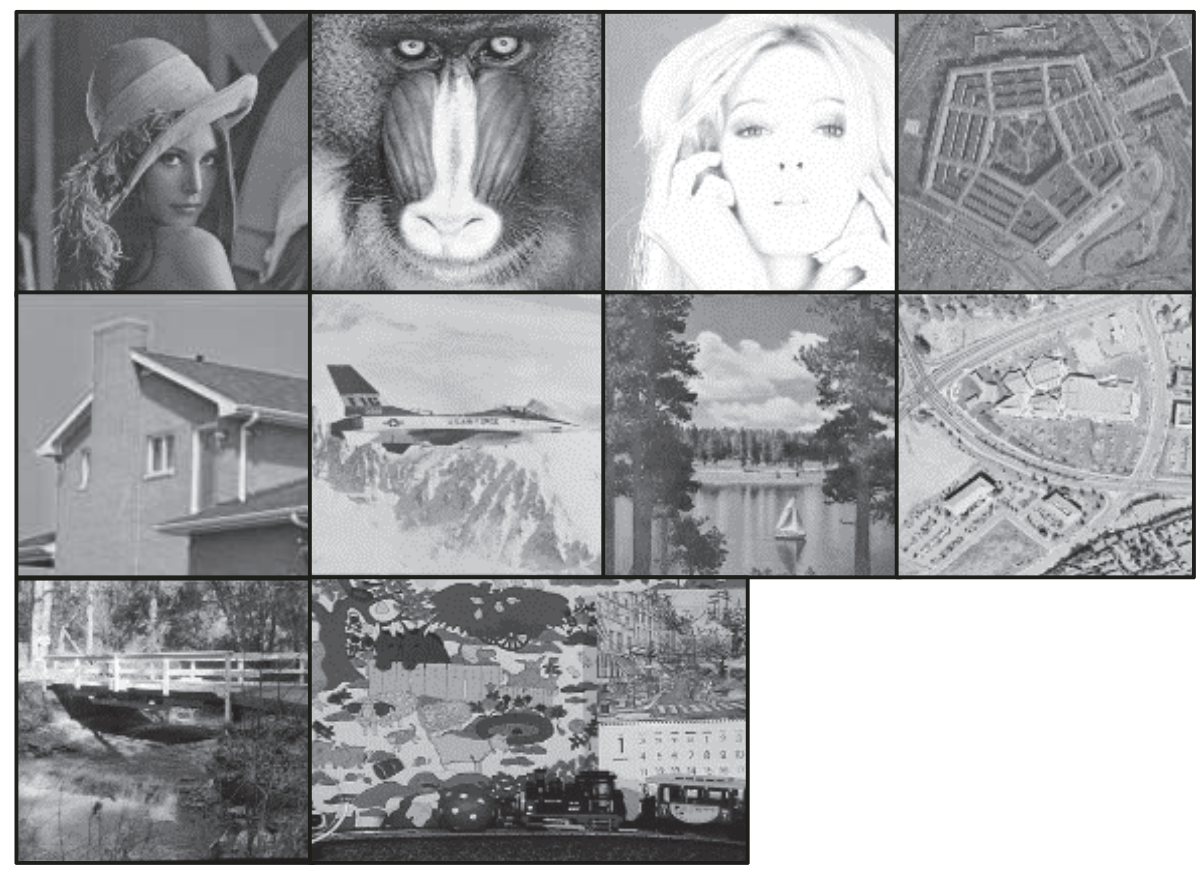

FIGURE 4: Ten standard testing images in the simulation.

$$
\begin{aligned}
& F_{G}(s, t)=e^{-\left(\left((i-s)^{2}+(j-t)^{2}\right) / 2 \sigma_{\mathrm{S}, \text { est }}^{2}\right)} \\
& F_{S R}(s, t)=e^{-\left(\left(I-x_{i+s, j+t}\right)^{2} / 2 \sigma_{\mathrm{R}, \mathrm{est}}^{2}\right)}
\end{aligned}
$$

where $N$ is the window size, $x$ is input pixel, $I=x_{i, j}$ for linear noise, $I=R M$ for nonlinear noise, $y_{i, j}=x_{i, j}$ for noise free, $s, t=-N$ to $N$, and $\sigma_{\text {R.est }}$ and $\sigma_{\text {S.est }}$ are radiometric weight and spatial weight, respectively.

\section{Simulation Results}

In this section, we first describe our learning process and simulate the performance of the proposed noise estimation technique. Second, we evaluate the performance of smart switching bilateral filter (SSBF) as compared to nonlocal sparse representation (NCSR), block-matching and 3D filtering (BM3D), trilateral filter (TF), traditional switching bilateral filter $(\mathrm{SBF})$, and optimum switching bilateral filter (OSBF). In our simulation, we consider four types of noise-Gaussian noise, salt-and-pepper noise, uniform impulse noise, and speckle noise. For Gaussian noise, we consider five levels of SNR-15, 20, 25, 30, and 35dB. Other noises consider five distribution values including 10, 20, 30, 40, and 50. For image types, we consider ten standard images including Lena (256x256), Baboon (256x256), Girl (256x256), Pentagon $(512 \times 512)$, House (128x128), Airplane (256x256), Sailboat (256x256), Aerial (256x256), Streamand-bridge (256x256), and Mobilcal frame $(352 \times 240)$ as shown in Figure 4. To evaluate the performance of filters, the performances of two of learned images-Lena (256x256) and Pentagon (512x512)-and nonlearning image-Pepper (256x256)-are simulated and illustrated in this paper.
5.1. Learning Process and Noise Estimation Evaluation. In this sub-section, we describe the learning process of our proposed estimated noise characterization scheme. To generate DW patterns in the knowledge base, ten standard images-Lena (256x256), Baboon (256x256), Girl (256x256), Pentagon (512x512), House (128x128), Airplane (256x256), Sailboat (256x256), Aerial (256x256), Stream-and-bridge (256x256), and Mobilcal frame (352x240) - are considered. These training images are contaminated with mixed noise between linear and nonlinear noise under different strengths of each type of noise where the strength is separated into four groups. The types of mixed noise images are as follows:

(i) Group A: Gaussian noise and salt-and-pepper noise

(ii) Group B: Gaussian noise and uniform impulse noise

(iii) Group C: speckle noise and salt-and-pepper noise

(iv) Group D: speckle noise and uniform impulse noise

Therefore, the simulated type of noisy image can be separated into four groups under different 16 levels of noise strength as shown in Table 2.

As mentioned earlier, in this paper, we first learned the DW patterns for four types of noise with 16 levels of noise strength. For the convenience of analyzing, as shown in Figure 5, we illustrate level 6 of each noise group because it is easy to distinguish the type of mixed noise. As depicted in the figure, each type of mixed noise has a specific DW pattern and is different from other types. As illustrated in the figure, Gaussian noise and speckle noise can be distinguished by the slope of the DW pattern at a low level, i.e., DW values from 0 to 10 . When the image is contaminated by Gaussian noise, the slope of the DW pattern at low level is high. This is because the differences between the considered pixel of the window 
TABLE 2: 16 levels of mixed noise of groups A, B, C, and D.

\begin{tabular}{|c|c|c|c|c|c|c|c|c|}
\hline \multirow{2}{*}{$\begin{array}{l}\text { group } \\
\text { Level }\end{array}$} & \multicolumn{2}{|c|}{ A. } & \multicolumn{2}{|c|}{ B. } & \multicolumn{2}{|c|}{ C. } & \multicolumn{2}{|c|}{ D. } \\
\hline & $\begin{array}{l}\text { Gaussian } \\
\text { (dB) }\end{array}$ & $\begin{array}{c}\text { Salt-and- } \\
\text { pepper } \\
(\rho)\end{array}$ & $\begin{array}{l}\text { Gaussian } \\
\text { (dB }(\end{array}$ & $\begin{array}{l}\text { Uniform } \\
\text { impulse }(\rho)\end{array}$ & Speckle $(\rho)$ & $\begin{array}{c}\text { Salt-and- } \\
\text { pepper } \\
(\rho)\end{array}$ & Speckle ) $\rho$ ) & $\begin{array}{l}\text { Uniform } \\
\text { impulse }(\rho)\end{array}$ \\
\hline 1 & 20 & 10 & 20 & 10 & 10 & 10 & 10 & 10 \\
\hline 2 & 20 & 20 & 20 & 20 & 10 & 20 & 10 & 20 \\
\hline 3 & 20 & 30 & 20 & 30 & 10 & 30 & 10 & 30 \\
\hline 4 & 20 & 40 & 20 & 40 & 10 & 40 & 10 & 40 \\
\hline 5 & 25 & 10 & 25 & 10 & 20 & 10 & 20 & 10 \\
\hline 6 & 25 & 20 & 25 & 20 & 20 & 20 & 20 & 20 \\
\hline 7 & 25 & 30 & 25 & 30 & 20 & 30 & 20 & 30 \\
\hline 8 & 25 & 40 & 25 & 40 & 20 & 40 & 20 & 40 \\
\hline 9 & 30 & 10 & 30 & 10 & 30 & 10 & 30 & 10 \\
\hline 10 & 30 & 20 & 30 & 20 & 30 & 20 & 30 & 20 \\
\hline 11 & 30 & 30 & 30 & 30 & 30 & 30 & 30 & 30 \\
\hline 12 & 30 & 40 & 30 & 40 & 30 & 40 & 30 & 40 \\
\hline 13 & 35 & 10 & 35 & 10 & 40 & 10 & 40 & 10 \\
\hline 14 & 35 & 20 & 35 & 20 & 40 & 20 & 40 & 20 \\
\hline 15 & 35 & 30 & 35 & 30 & 40 & 30 & 40 & 30 \\
\hline 16 & 35 & 40 & 35 & 40 & 40 & 40 & 40 & 40 \\
\hline
\end{tabular}

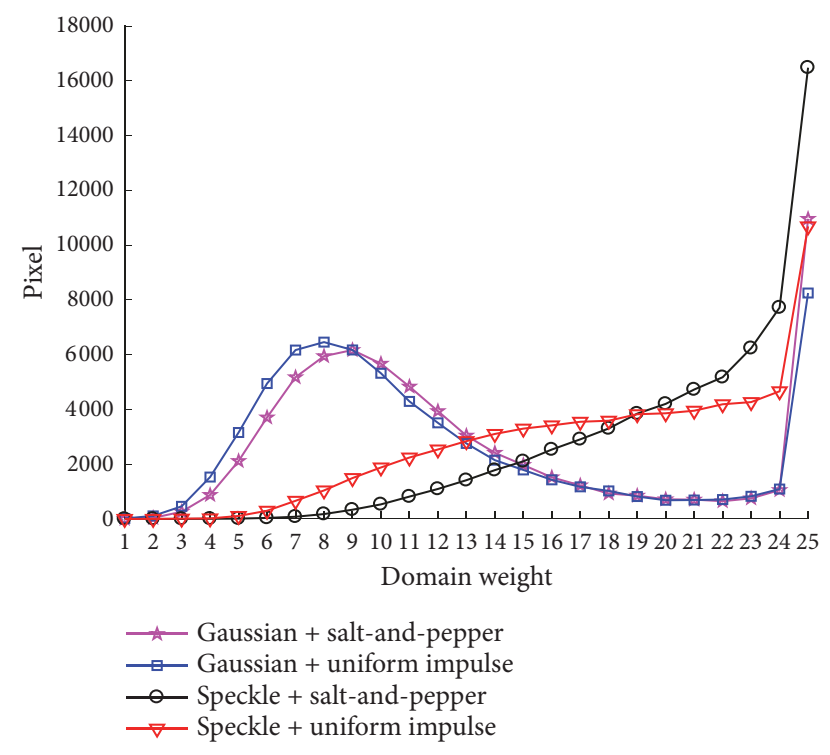

FIGURE 5: DW pattern for four groups of mixed noise.

and its neighbors are small because Gaussian noise corrupts the image on a normal distribution pattern by increasing or decreasing the intensity of the pixel. If the image is corrupted by speckle noise, the slope of the DW pattern at low level is low. It can be noticed that the slope of the DW pattern of the image contaminated by speckle noise increases at the level of DW value which is greater than 7. For a nonlinear noise, the type of noise can be distinguished by determining the DW value of level 25. It can be noticed that the DW value at the $25^{\text {th }}$ level when the image is contaminated by saltand-pepper is higher than uniform impulse noise. Then, to estimate the type of mixed noise, we calculate the normalized mean-squared error (Normalize MSE) between DW pattern of detected image and learned DW pattern. The Normalize MSE is given as

$$
\begin{aligned}
& \text { Normalize MSE } \\
& \quad=\frac{\sum_{L V=1}^{G N}\left(D W P_{L V} /(C * R)\right)-D W R_{L V} /(C * R)}{G N}
\end{aligned}
$$

when $G N$ is group number $=25, D W P$ is domain weight pattern, $D W \mathrm{R}$ is domain weight of received image, and $C$ and $\mathrm{R}$ are size of image.

Second, we evaluate the performance of the proposed estimation technique for estimating the type of mixed noise and strength. The performance of an estimation is determined by the mean-squared error (MSE) of a noisy training image and noisy test image where the MSE can be calculated by

$$
M S E=\frac{1}{n} \sum_{i=1}^{n}\left(x_{i}-y_{i}\right)^{2}
$$

where $x_{i}$ and $y_{i}$ represent the DW of noisy training image and DW of noisy test image, respectively.

The SSIM can be formulated by

$$
\operatorname{SSIM}(i, j)=l(i, j) c(i, j) s(i, j)
$$

where $l(i, j)$ is the luminance comparison function, $c(i, j)$ is the contrast comparison function, and $s(i, j)$ is the structure comparison function. 

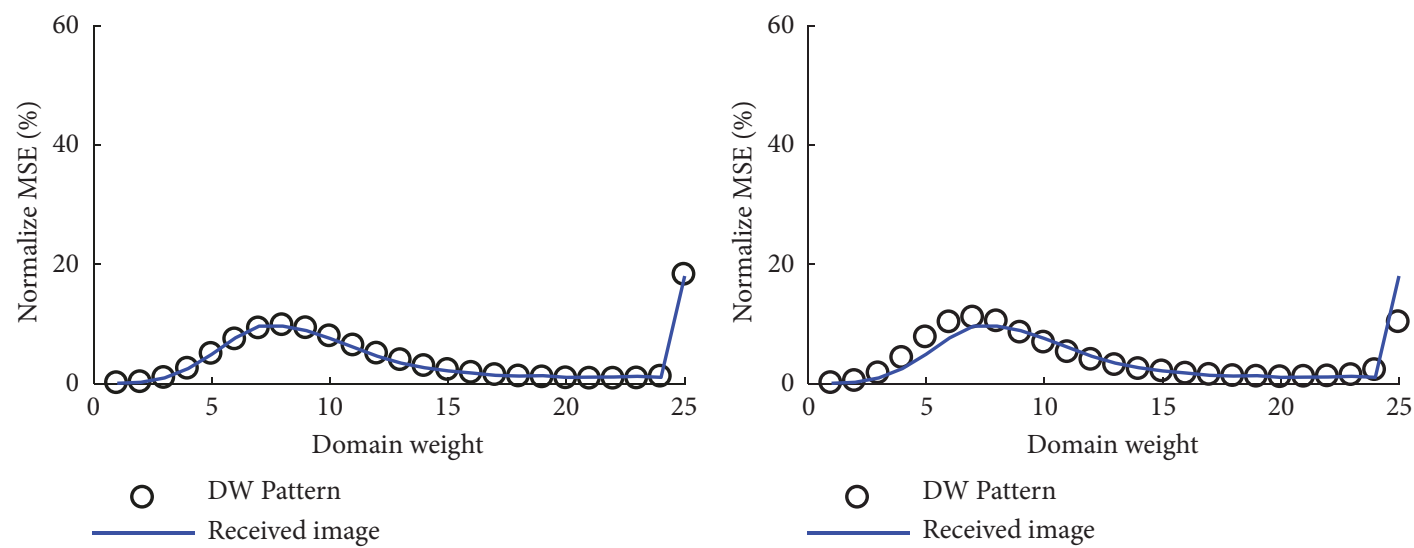

(a)

(b)

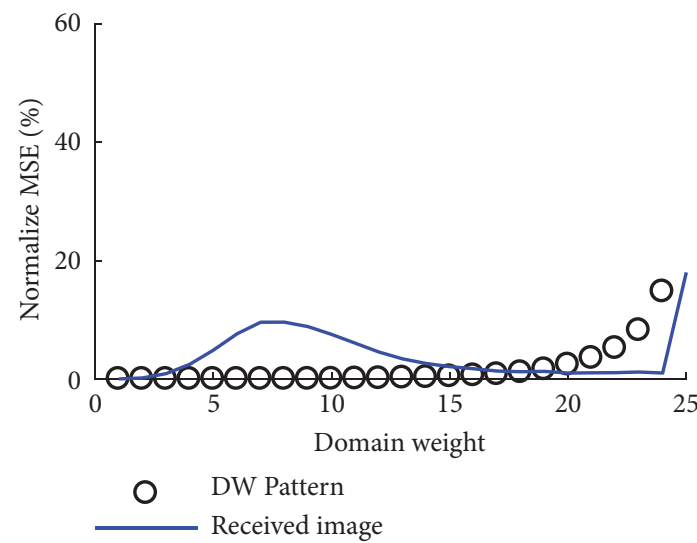

(c)

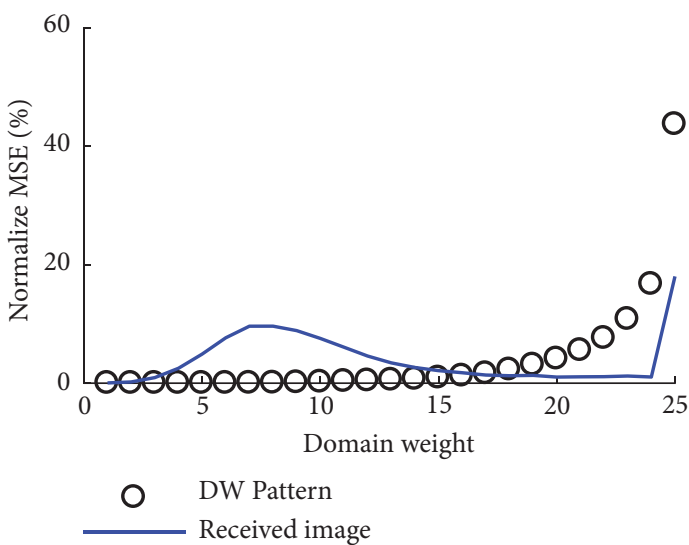

(d)

FIGURE 6: Comparison of detected group A mixed noise and the four patterns in knowledge base: (a) Gaussian mixed with salt-and-pepper noise, (b) Gaussian mixed with uniform impulse noise, (c) speckle mixed with salt-and-pepper, and (d) speckle mixed with uniform impulse noise.

TABLE 3: Normalize MSE of estimated noise type in Gaussian (25dB) mixed with salt-and-pepper (20\%) of received image from Figure 6.

\begin{tabular}{lc}
\hline Noise type & Normalize MSE \\
\hline Gaussian mix salt \& pepper & 0.0622 \\
Gaussian mix uniform impulse & 3.3854 \\
Speckle mix salt \& pepper & 101.7631 \\
Speckle mix uniform impulse & 61.6393 \\
\hline
\end{tabular}

In Figure 6, the results of noise type estimation when the received image is corrupted by Gaussian noise of $25 \mathrm{~dB}$ and salt-and-pepper of $20 \%$ are illustrated. It can be noticed that the DW pattern of received image is near to the DW pattern of groups $\mathrm{A}$ and $\mathrm{B}$. However, we can distinguish between groups $\mathrm{A}$ and $\mathrm{B}$ by determining the DW value of the $25^{\text {th }}$ group. The salt-and-pepper noise affects the changing of pixel's intensity into definitely white or black while the uniform noise affects the changing of pixel's intensity to dark white or light black. The results of MSE are shown in Table 3. Therefore, it can be identified that the received image is corrupted by Gaussian noise mixed with salt-and-pepper noise.

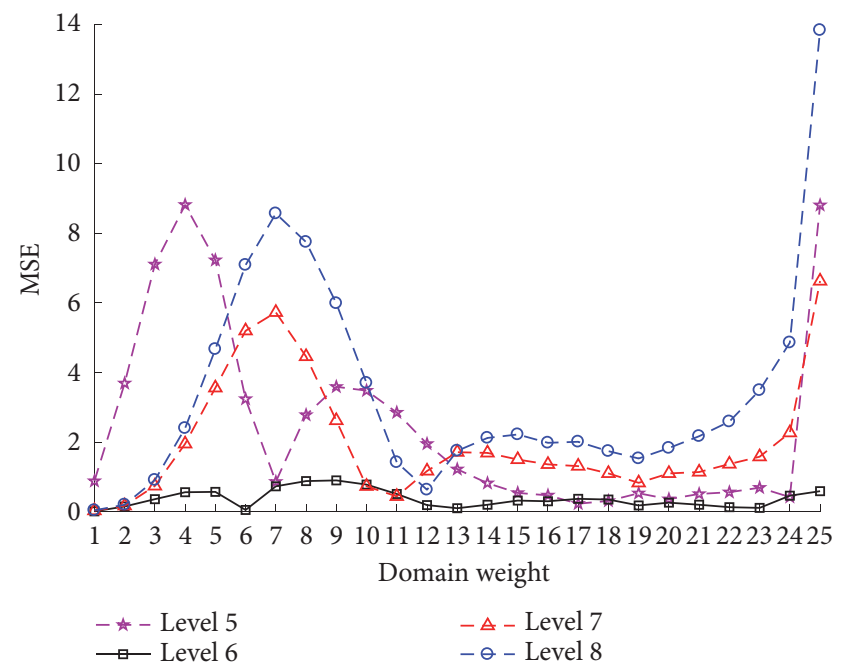

FIGURE 7: Comparison of MSE for group A mixed noise under 4 different levels of mixed noise strength.

Then, the DW pattern of received image is analyzed comprehensively by comparing to the DW patterns in group A to determine the strength of noises. As depicted in Figure 7, 

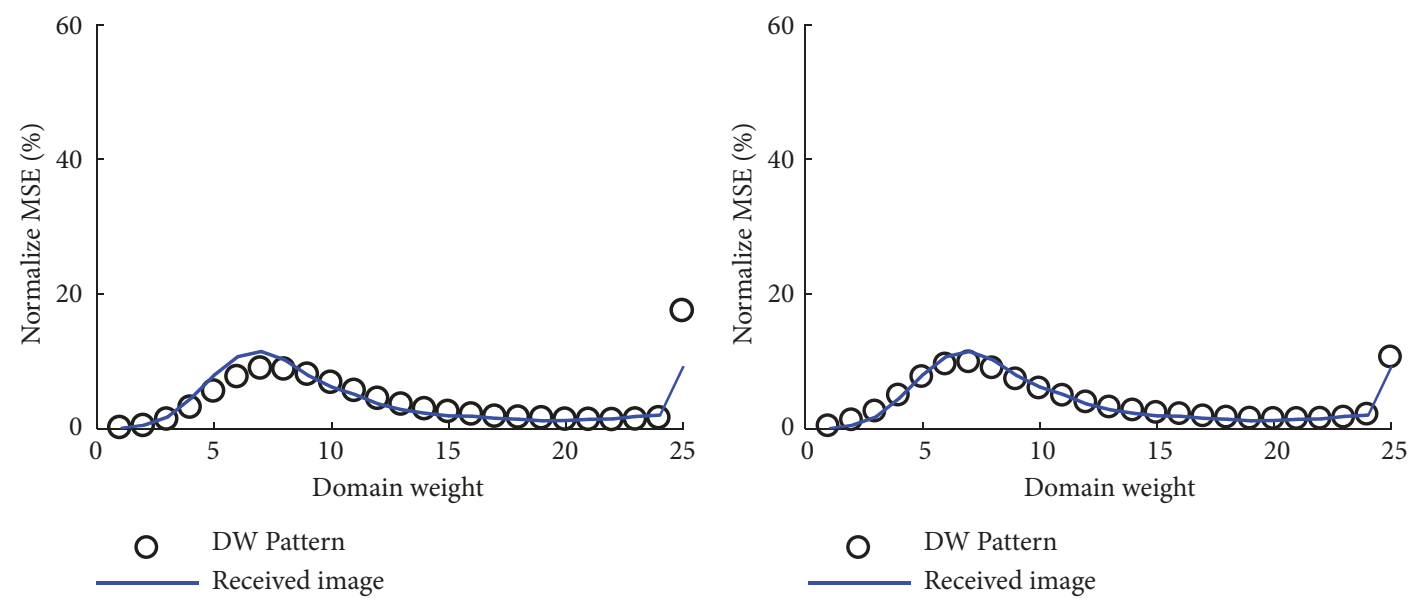

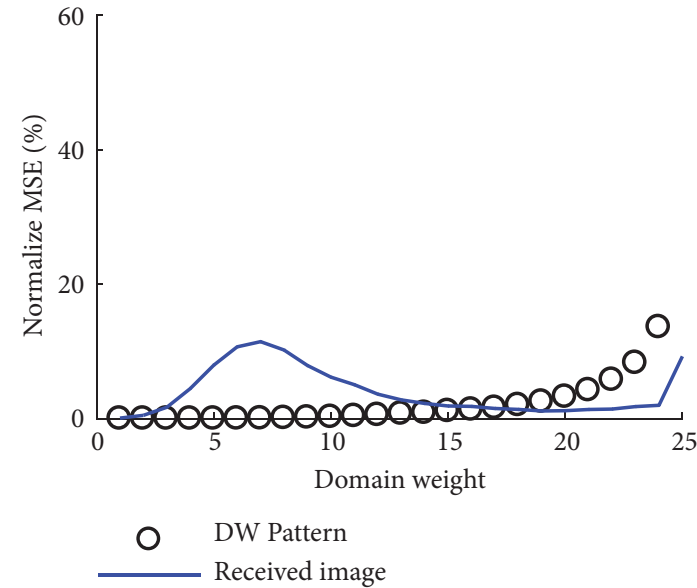

(c)

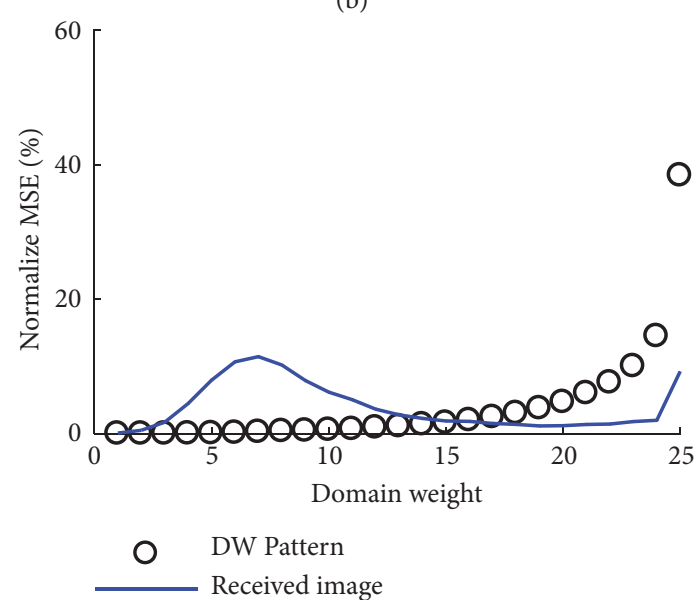

(d)

Figure 8: Comparison of detected group B mixed noise and the four patterns in knowledge base: (a) Gaussian mixed with salt-and-pepper noise, (b) Gaussian mixed with uniform impulse noise, (c) speckle mixed with salt-and-pepper, and (d) speckle mixed with uniform impulse noise.

we can see the MSE between the DW patterns of received image and the DW patterns of levels 5, 6, 7, and 8 of group A. As shown in Table 4, the MSE of the DW pattern of the received image and level 6 is the lowest, and the SSIM is the highest. It means that the detected pattern of received image matches level 6 . Therefore, the detected levels of noise strength are $25 \mathrm{~dB}$ of Gaussian noise and salt-and-pepper with $\rho$ equal to $20 \%$. Then, the estimate radiometric weight $\left(\sigma_{\text {R.est }}\right)$ and spatial weight $\left(\sigma_{\text {S.est }}\right)$ can be selected from the learning-radiometric weight $\left(\sigma_{\mathrm{R}}\right)$ and spatial weight $\left(\sigma_{\mathrm{S}}\right)$ table, which is shown in Table 5. To learn the $\sigma_{\mathrm{S}}$ and $\sigma_{\mathrm{R}}$ for Table 5 , we investigate $\sigma_{\mathrm{S}}$ and $\sigma_{\mathrm{R}}$ which achieve the optimal performance of 4 standard images and these optimal values are averaged for each level of noise strength.

The estimation of mixed noise groups $\mathrm{B}, \mathrm{C}$, and $\mathrm{D}$ is illustrated in Figures 8, 9, and 10, respectively. As described earlier, the linear noise can be estimated by using DW values at the lower range (3 to 10). For the nonlinear noise type, the $25^{\text {th }}$ value of DW can be used to identify the noise. As shown in Table 6, the performance of noise type and strength estimation is depicted. By estimating the noise type and strength using DW pattern, the proposed estimated noise
TABLE 4: Compare MSE and SSIM values of noisy image for level 6 (Gaussian 25dB mixed with salt-and-pepper 20\%).

\begin{tabular}{lcc}
\hline Level & MSE & SSIM \\
\hline 1 & 23.47 & 0.7612 \\
2 & 45.34 & 0.6324 \\
3 & 86.67 & 0.5678 \\
4 & 105.62 & 0.4832 \\
5 & 61.96 & 0.6015 \\
6 & 9.26 & 0.8746 \\
7 & 50.40 & 0.6145 \\
8 & 85.51 & 0.5753 \\
9 & 85.16 & 0.5764 \\
10 & 25.16 & 0.7565 \\
11 & 33.68 & 0.7378 \\
12 & 73.13 & 0.5846 \\
13 & 92.04 & 0.5246 \\
14 & 32.99 & 0.7414 \\
15 & 29.22 & 0.7432 \\
16 & 67.51 & 0.5921 \\
\hline
\end{tabular}




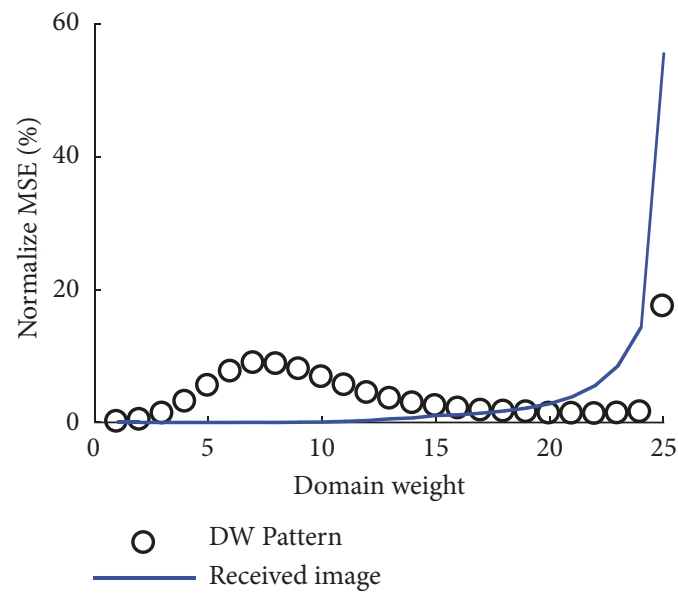

(a)

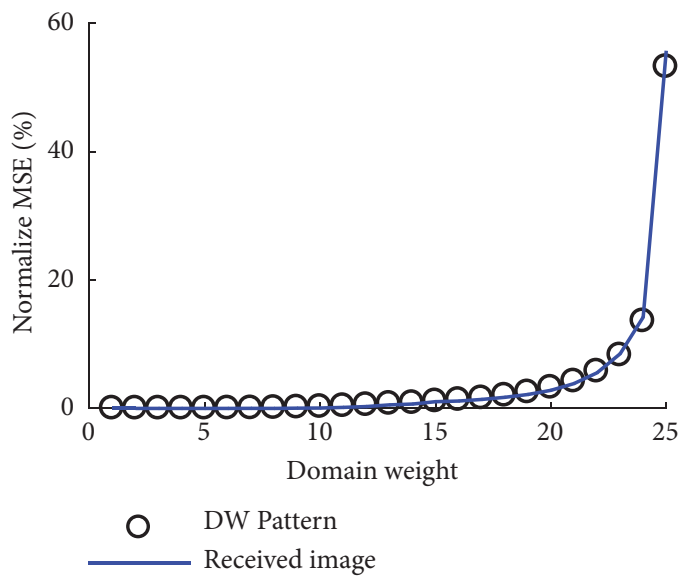

(c)

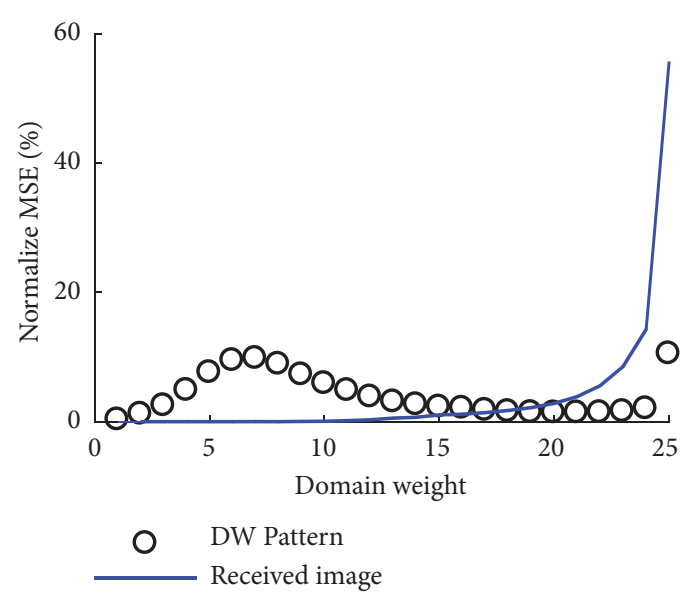

(b)

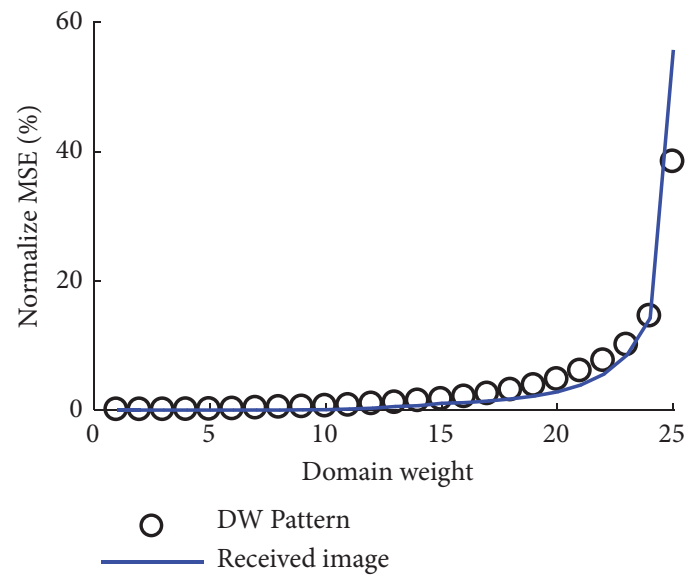

(d)

Figure 9: Comparison of detected group C mixed noise and the four patterns in knowledge base: (a) Gaussian mixed with salt-and-pepper noise, (b) Gaussian mixed with uniform impulse noise, (c) speckle mixed with salt-and-pepper, and (d) speckle mixed with uniform impulse noise.

TABLE 5: The values of $\sigma_{\text {R.est }}$ and $\sigma_{\text {S.est }}$ of (a) Gaussian noise environment and (b) salt-and-pepper noise, uniform impulse noise, and speckle noise environments.

(a)

\begin{tabular}{|c|c|c|c|c|c|c|}
\hline \multirow{2}{*}{ Noise } & & \multicolumn{5}{|c|}{ SNR } \\
\hline & & $\begin{array}{l}15 \\
\mathrm{~dB}\end{array}$ & $20 \mathrm{~dB}$ & $25 \mathrm{~dB}$ & $\begin{array}{l}30 \\
\mathrm{~dB}\end{array}$ & $35 \mathrm{~dB}$ \\
\hline \multirow{2}{*}{ Gaussian } & $\sigma_{\text {R.est }}$ & 59 & 36 & 16 & 6 & 3 \\
\hline & $\sigma_{\text {S.est }}$ & 99 & 90 & 88 & 95 & 93 \\
\hline
\end{tabular}

(b)

\begin{tabular}{lcccccc}
\hline \multirow{2}{*}{ Noise } & & \multicolumn{5}{c}{$\rho$} \\
& & $10 \%$ & $20 \%$ & $30 \%$ & $40 \%$ & $50 \%$ \\
\hline \multirow{2}{*}{ Salt-and-pepper } & $\sigma_{\text {R.est }}$ & 71 & 56 & 56 & 52 & 51 \\
& $\sigma_{\text {S.est }}$ & 90 & 85 & 94 & 87 & 90 \\
\hline \multirow{2}{*}{ Uniform impulse } & $\sigma_{\text {R.est }}$ & 58 & 42 & 32 & 34 & 44 \\
& $\sigma_{\text {S.est }}$ & 99 & 95 & 88 & 93 & 93 \\
\hline \multirow{2}{*}{ Speckle } & $\sigma_{\text {R.est }}$ & 198 & 263 & 270 & 265 & 272 \\
& $\sigma_{\text {S.est }}$ & 246 & 286 & 247 & 275 & 288 \\
\hline
\end{tabular}

TABLE 6: A performance of noise type and strength estimation.

\begin{tabular}{lcccc}
\hline Level & Group A & Group B & Group C & Group D \\
\hline 1 & $95 \%$ & $94 \%$ & $96 \%$ & $94 \%$ \\
2 & $85 \%$ & $84 \%$ & $86 \%$ & $83 \%$ \\
3 & $75 \%$ & $73 \%$ & $76 \%$ & $75 \%$ \\
4 & $65 \%$ & $64 \%$ & $73 \%$ & $72 \%$ \\
5 & $96 \%$ & $95 \%$ & $94 \%$ & $93 \%$ \\
6 & $95 \%$ & $93 \%$ & $83 \%$ & $82 \%$ \\
7 & $94 \%$ & $92 \%$ & $83 \%$ & $82 \%$ \\
8 & $85 \%$ & $82 \%$ & $75 \%$ & $73 \%$ \\
9 & $94 \%$ & $93 \%$ & $92 \%$ & $91 \%$ \\
10 & $93 \%$ & $92 \%$ & $85 \%$ & $84 \%$ \\
11 & $83 \%$ & $82 \%$ & $81 \%$ & $80 \%$ \\
12 & $74 \%$ & $72 \%$ & $73 \%$ & $72 \%$ \\
13 & $98 \%$ & $96 \%$ & $85 \%$ & $83 \%$ \\
14 & $96 \%$ & $94 \%$ & $80 \%$ & $79 \%$ \\
15 & $94 \%$ & $92 \%$ & $75 \%$ & $74 \%$ \\
16 & $92 \%$ & $90 \%$ & $67 \%$ & $65 \%$ \\
Average & $88 \%$ & $87 \%$ & $82 \%$ & $80 \%$ \\
\hline
\end{tabular}




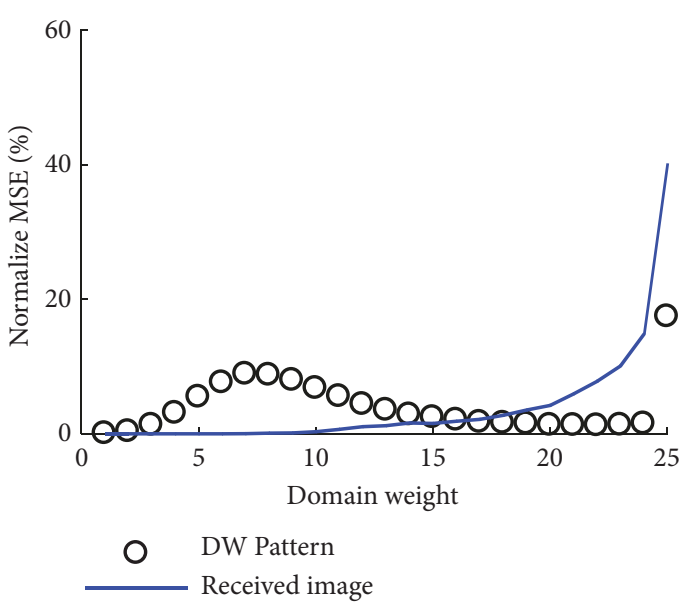

(a)

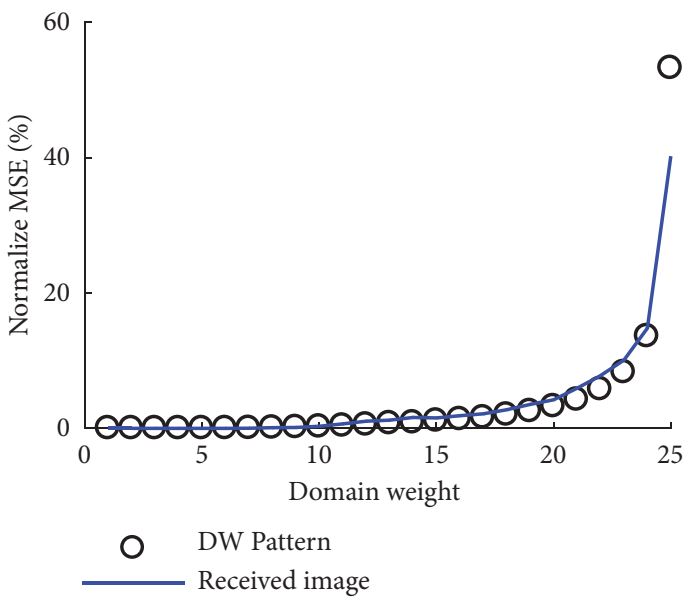

(c)

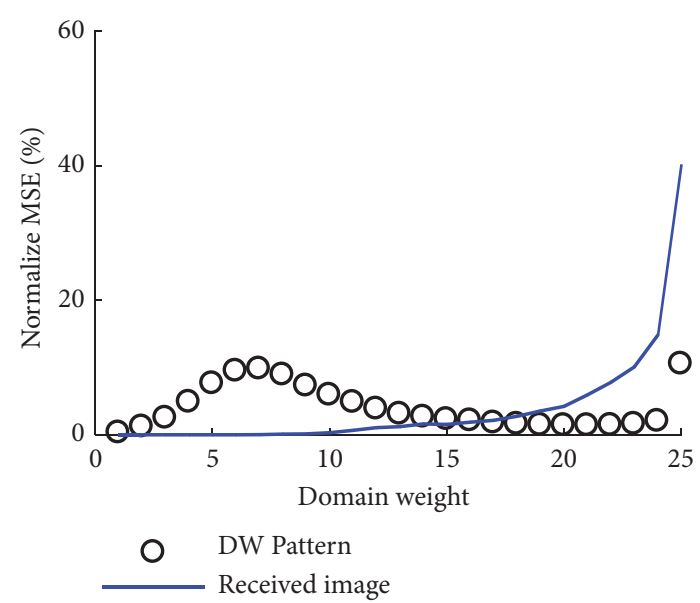

(b)

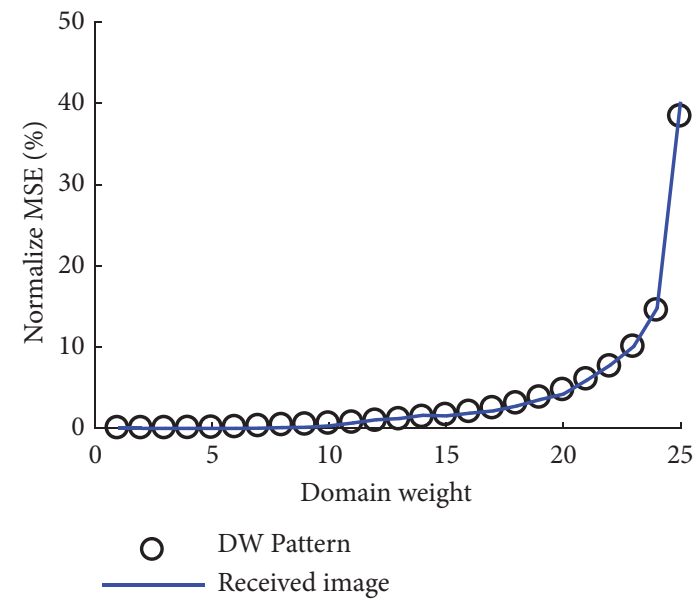

(d)

FIGURE 10: Comparison of detected group D mixed noise and the four patterns in knowledge base: (a) Gaussian mixed with salt-and-pepper noise, (b) Gaussian mixed with uniform impulse noise, (c) speckle mixed with salt-and-pepper, and (d) speckle mixed with uniform impulse noise.

characterization scheme gives high estimation performance as $80 \%$.

5.2. Simulation Results. In this subsection, we simulate the performance of smart switching bilateral filter (SSBF) as compared to 5 filters-nonlocal sparse representation (NCSR), block-matching and 3D filtering (BM3D), trilateral filter (TF), traditional switching bilateral filter (SBF), and optimum switching bilateral filter (OSBF) - for 4 types of mixed noise under 16 levels of noise strength. To determine the type of noise at the determined pixel, $\mathrm{T}_{\mathrm{RM}}$ is set to be 40 . The spatial weight $\left(\sigma_{\mathrm{S}}\right)$ and radiometric weight $\left(\sigma_{\mathrm{R}}\right)$ for traditional $\mathrm{SBF}$ are set as $\sigma_{\mathrm{S}}$ which is set to 3 and $\sigma_{\mathrm{R}}$ which is set to 0.5 for linear noise. For nonlinear noise, $\sigma_{\mathrm{S}}$ is set to 5 and $\sigma_{\mathrm{R}}$ is set to 0.3 [22]. For OSBF, $\sigma_{\mathrm{S}}$ and $\sigma_{\mathrm{R}}$ which are set on the assumption of noise strength are exactly known.

As shown in Figure 11, the performances of NCSR, BM3D, TF, SBF, OSBF, and SSBF are compared when the image is corrupted by the Gaussian noise and salt-and-pepper noise under 16 levels of mixed noise strength. Once the mixed noise strength is exactly known, OSBF gives the best mixed noise removal performance as compared to others. However, the OSBF is challenging to be implemented in practice because it is difficult to be known as the exact noise type and strength. On the other hand, SBF gives low performance because the values of $\sigma_{\mathrm{S}}$ and $\sigma_{\mathrm{R}}$ are not appropriate to the noise type and strength. From Table 7, NCSR and TF show nearly the same performance on removing corrupted Gaussian noise mixed with salt-and-pepper noise. However, their performance is worse than the proposed SSBF filter. Moreover, the performance of NCSR and TF noticeably drops when noise strength increases. Even if BM3D works well on denoising the Gaussian noise, its performance becomes worse when Gaussian noise mixes with others. Therefore, we consider performance of OSBF as the upper boundary. As depicted in the figure, SSBF gives high performance nearly to the upper benchmark without any knowledge of noise type and strength. By estimating the noise type and strength using the proposed estimated noise characterization scheme, the $\sigma_{\text {S.est }}$ and $\sigma_{\text {R.est }}$ are selected on the detected noise type and strength. Therefore, SSBF gives high performance since $\sigma_{\text {S.est }}$ and $\sigma_{\text {R.est }}$ are appropriate. 


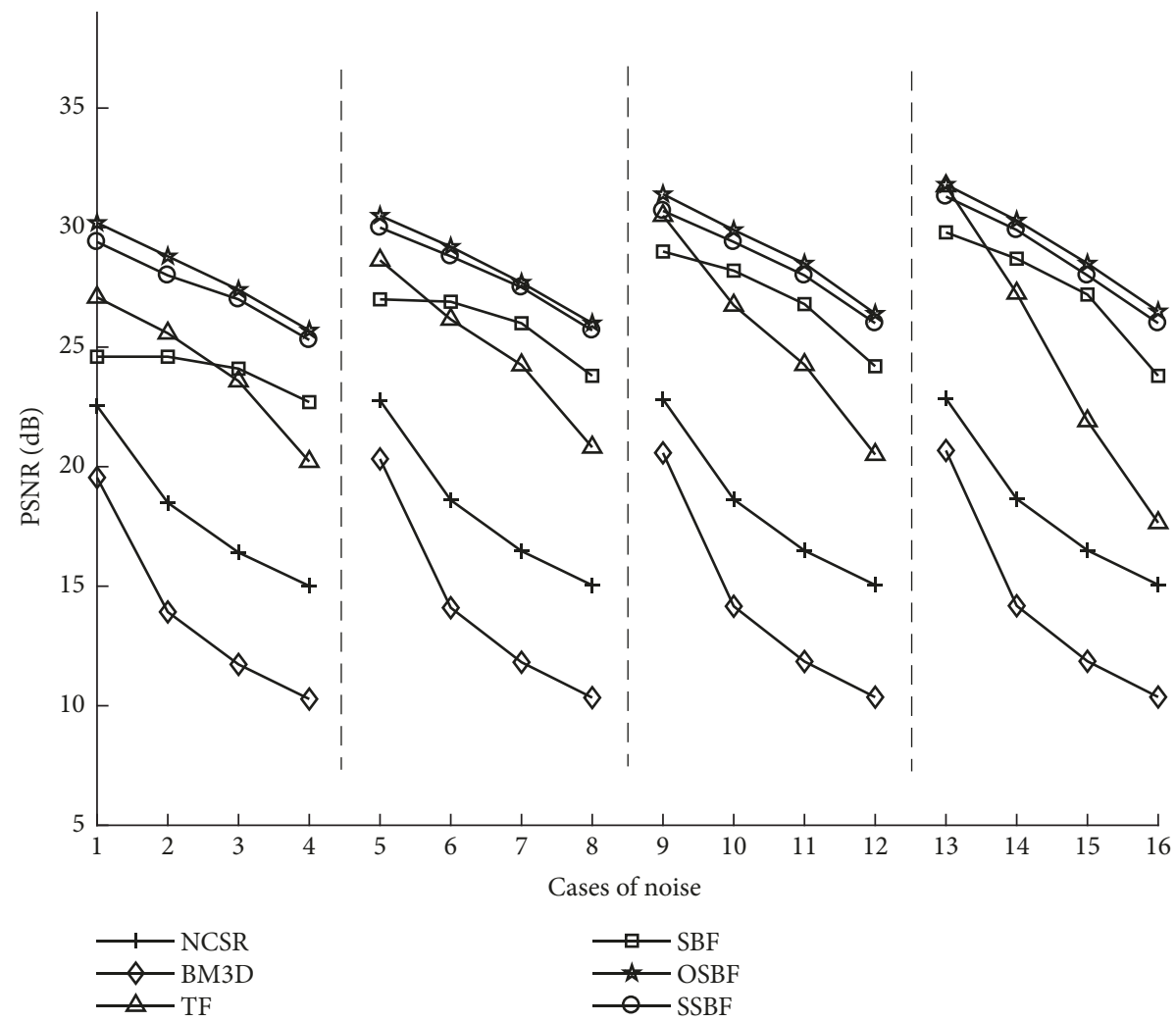

FIGURE 11: PSNR comparison of NCSR, BM3D, TF, SBF, OSBF, and SSBF for image that is corrupted Gaussian noise mixed with salt-andpepper noise.

TABLE 7: PSNR comparison of NCSR, BM3D, TF, SBF, OSBF, and SSBF for image that is corrupted Gaussian noise mixed with saltand-pepper noise.

\begin{tabular}{lcccccc}
\hline Level & NCSR & BM3D & TF & SBF & OSBF & SSBF \\
\hline 1 & 22.54 & 16.35 & 22.46 & 20.83 & 27.21 & 24.55 \\
2 & 18.49 & 14.33 & 20.22 & 20.62 & 25.22 & 23.55 \\
3 & 16.41 & 13.06 & 17.25 & 20.12 & 23.50 & 22.11 \\
4 & 15.01 & 12.08 & 14.61 & 19.05 & 21.50 & 20.10 \\
5 & 22.77 & 13.44 & 19.34 & 19.04 & 24.50 & 23.96 \\
6 & 18.60 & 12.46 & 16.53 & 18.44 & 23.00 & 22.60 \\
7 & 16.47 & 12.10 & 14.67 & 17.54 & 22.00 & 21.52 \\
8 & 15.04 & 11.13 & 12.36 & 16.26 & 21.40 & 20.40 \\
9 & 22.82 & 11.87 & 17.34 & 17.66 & 23.80 & 22.83 \\
10 & 18.63 & 11.31 & 14.63 & 16.93 & 22.50 & 21.85 \\
11 & 16.49 & 10.84 & 12.53 & 15.84 & 21.30 & 20.91 \\
12 & 15.05 & 10.43 & 11.14 & 14.66 & 20.20 & 19.10 \\
13 & 22.85 & 10.87 & 15.97 & 16.75 & 23.60 & 22.89 \\
14 & 18.65 & 10.50 & 14.00 & 15.85 & 22.50 & 21.89 \\
15 & 16.50 & 10.20 & 11.51 & 14.77 & 21.40 & 20.46 \\
16 & 15.06 & 09.92 & 10.43 & 13.61 & 19.70 & 18.65 \\
\hline
\end{tabular}

As shown in Figure 12, the performance comparison of NCSR, BM3D, TF, SBF, OSBF, and SSBF when image is corrupted by Gaussian mixed with uniform impulse noise is depicted. When the noise is mixed by Gaussian noise, SSBF
TABLE 8: PSNR comparison of NCSR, BM3D, TF, SBF, OSBF, and SSBF for image that is corrupted Gaussian noise mixed with uniform impulse noise.

\begin{tabular}{lcccccc}
\hline Level & NCSR & BM3D & TF & SBF & OSBF & SSBF \\
\hline 1 & 24.51 & 24.00 & 27.12 & 26.95 & 28.91 & 28.22 \\
2 & 20.76 & 18.16 & 25.72 & 26.35 & 28.08 & 27.50 \\
3 & 18.69 & 15.33 & 23.84 & 24.22 & 26.53 & 26.00 \\
4 & 17.21 & 13.62 & 20.05 & 21.43 & 24.35 & 23.04 \\
5 & 24.76 & 24.42 & 28.75 & 28.12 & 30.54 & 29.44 \\
6 & 20.88 & 18.40 & 26.26 & 27.06 & 29.42 & 28.77 \\
7 & 18.73 & 15.41 & 24.46 & 25.35 & 26.61 & 26.33 \\
8 & 17.20 & 13.62 & 20.51 & 22.26 & 24.34 & 23.56 \\
9 & 24.79 & 24.45 & 30.06 & 28.22 & 32.03 & 31.11 \\
10 & 20.88 & 18.43 & 26.79 & 27.55 & 30.11 & 29.33 \\
11 & 18.71 & 15.39 & 24.53 & 26.45 & 28.43 & 27.45 \\
12 & 17.17 & 13.58 & 20.37 & 22.57 & 26.50 & 24.50 \\
13 & 24.73 & 24.33 & 31.08 & 28.55 & 32.86 & 31.22 \\
14 & 20.84 & 18.34 & 27.03 & 26.67 & 30.51 & 29.33 \\
15 & 18.66 & 15.32 & 23.64 & 24.33 & 28.94 & 27.26 \\
16 & 17.11 & 13.50 & 20.43 & 22.61 & 24.80 & 24.35 \\
\hline
\end{tabular}

can identify the type of mixed noise and its strength accurately. Therefore, the selected $\sigma_{\text {S.est }}$ and $\sigma_{\text {R.est }}$ are appropriate for the received image. Then, the performance of SSBF is near to OSBF. From Table 8, the performance of BM3D becomes 


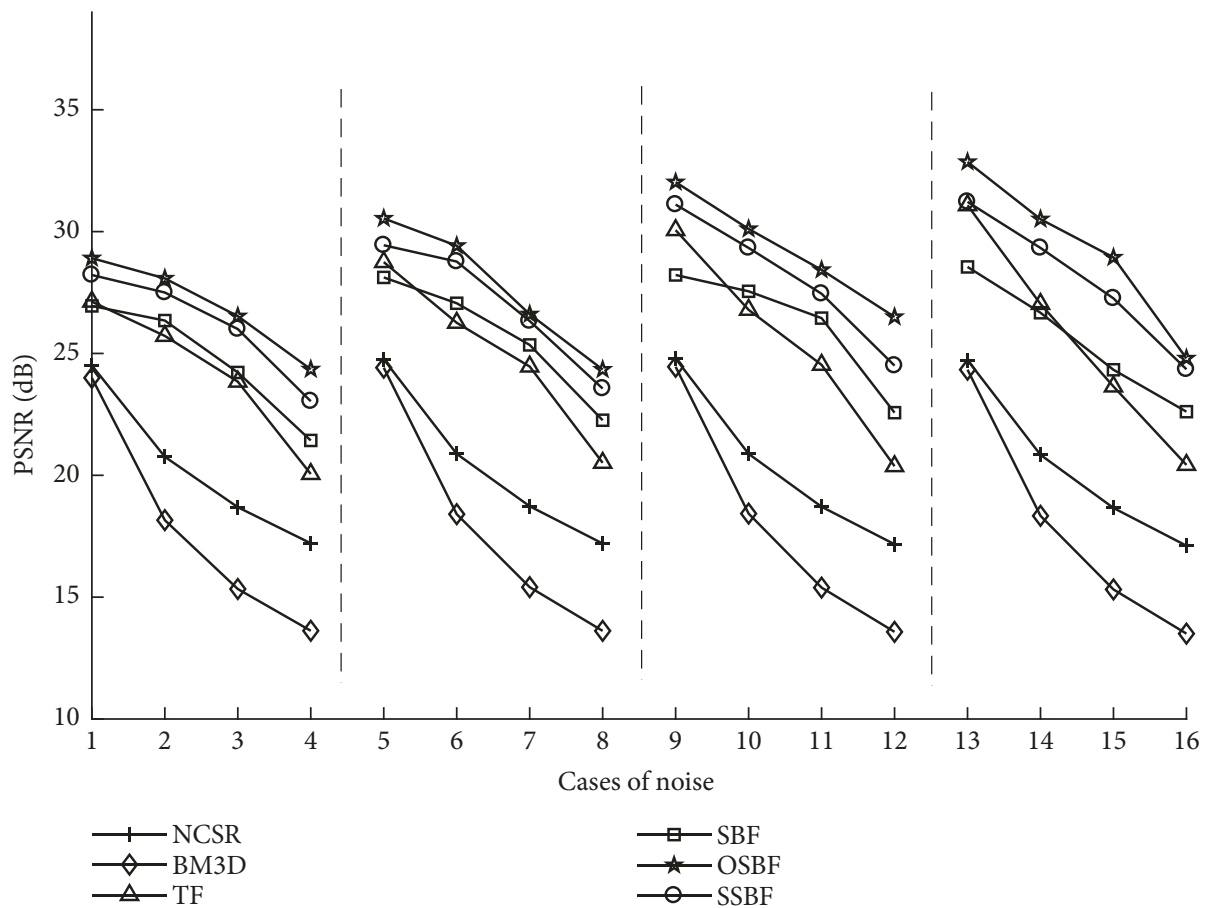

FIGURE 12: PSNR comparison of NCSR, BM3D, TF, SBF, OSBF, and SSBF for image that is corrupted Gaussian noise mixed with uniform impulse noise.

TABLE 9: PSNR comparison of NCSR, BM3D, TF, SBF, OSBF, and SSBF for image that is speckle noise mixed with salt-and-pepper noise.

\begin{tabular}{lcccccc}
\hline Level & NCSR & BM3D & TF & SBF & OSBF & SSBF \\
\hline 1 & 19.25 & 14.63 & 22.37 & 19.23 & 29.70 & 28.70 \\
2 & 16.88 & 12.23 & 20.61 & 18.75 & 28.70 & 27.50 \\
3 & 15.39 & 10.74 & 18.18 & 17.93 & 26.70 & 25.00 \\
4 & 14.27 & 09.63 & 16.03 & 16.59 & 25.10 & 23.50 \\
5 & 17.43 & 12.48 & 19.48 & 17.60 & 28.50 & 26.20 \\
6 & 15.91 & 11.07 & 17.59 & 16.85 & 26.50 & 24.80 \\
7 & 14.72 & 09.94 & 15.41 & 15.92 & 24.23 & 22.70 \\
8 & 13.83 & 09.09 & 13.82 & 14.64 & 22.10 & 20.83 \\
9 & 16.29 & 11.19 & 17.53 & 15.76 & 26.20 & 24.07 \\
10 & 15.12 & 10.16 & 15.80 & 15.24 & 25.10 & 23.05 \\
11 & 14.22 & 09.34 & 13.85 & 15.00 & 24.20 & 22.10 \\
12 & 13.50 & 08.66 & 13.48 & 14.45 & 22.60 & 20.80 \\
13 & 15.42 & 10.79 & 16.14 & 15.20 & 25.44 & 23.64 \\
14 & 14.36 & 09.86 & 14.67 & 14.80 & 23.22 & 22.11 \\
15 & 13.66 & 09.12 & 13.25 & 13.99 & 21.87 & 20.32 \\
16 & 13.24 & 08.49 & 12.62 & 12.90 & 20.25 & 19.32 \\
\hline
\end{tabular}

better when Gaussian noise mixes with uniform impulse noise. However, it still gives the worst mixed noise removal performance. SBF, TF, SSBF, and OSBF show much better performance than NCSR corrupted Gaussian noise mixed with uniform impulse noise environments.

When the noise is mixed by speckle noise as shown in Figures 13 and 14 and Tables 9 and 10, the performance of the SSBF is still near to OSBF since the type and strength of noise
TABLE 10: PSNR comparison of NCSR, BM3D, TF, SBF, OSBF, and SSBF for image that is speckle noise mixed with uniform impulse noise.

\begin{tabular}{lcccccc}
\hline Level & NCSR & BM3D & TF & SBF & OSBF & SSBF \\
\hline 1 & 20.45 & 16.35 & 22.46 & 20.83 & 27.21 & 24.55 \\
2 & 18.49 & 14.33 & 20.22 & 20.62 & 25.22 & 23.55 \\
3 & 17.22 & 13.06 & 17.25 & 20.12 & 23.50 & 22.11 \\
4 & 16.18 & 12.08 & 14.61 & 19.05 & 21.50 & 20.10 \\
5 & 18.26 & 13.44 & 19.34 & 19.04 & 24.50 & 23.96 \\
6 & 17.08 & 12.46 & 16.53 & 18.44 & 23.00 & 22.60 \\
7 & 16.92 & 12.10 & 14.67 & 17.54 & 22.00 & 21.52 \\
8 & 15.50 & 11.13 & 12.36 & 16.26 & 21.40 & 20.40 \\
9 & 16.90 & 11.87 & 17.34 & 17.66 & 23.80 & 22.83 \\
10 & 16.11 & 11.31 & 14.63 & 16.93 & 22.50 & 21.85 \\
11 & 15.51 & 10.84 & 12.53 & 15.84 & 21.30 & 20.91 \\
12 & 14.96 & 10.43 & 11.14 & 14.66 & 20.20 & 19.10 \\
13 & 15.94 & 10.87 & 15.97 & 16.75 & 23.60 & 22.89 \\
14 & 15.70 & 10.50 & 14.00 & 15.85 & 22.50 & 21.89 \\
15 & 15.18 & 10.20 & 11.51 & 14.77 & 21.40 & 20.46 \\
16 & 14.69 & 09.92 & 10.43 & 13.61 & 19.70 & 18.65 \\
\hline
\end{tabular}

are efficiently estimated. It can be seen that since the proposed estimated noise characterization scheme analyzes mixed noise by determining the distribution pattern of domain values (DW) instead of using the histogram, the proposed technique distinguishes the type and strength of mixed noise accurately. Since histogram shows the distribution of pixels' intensity, the type of mixed noise cannot be distinguished. On the other hand, NCSR, BM3D, TF, and SBF are not proposed 


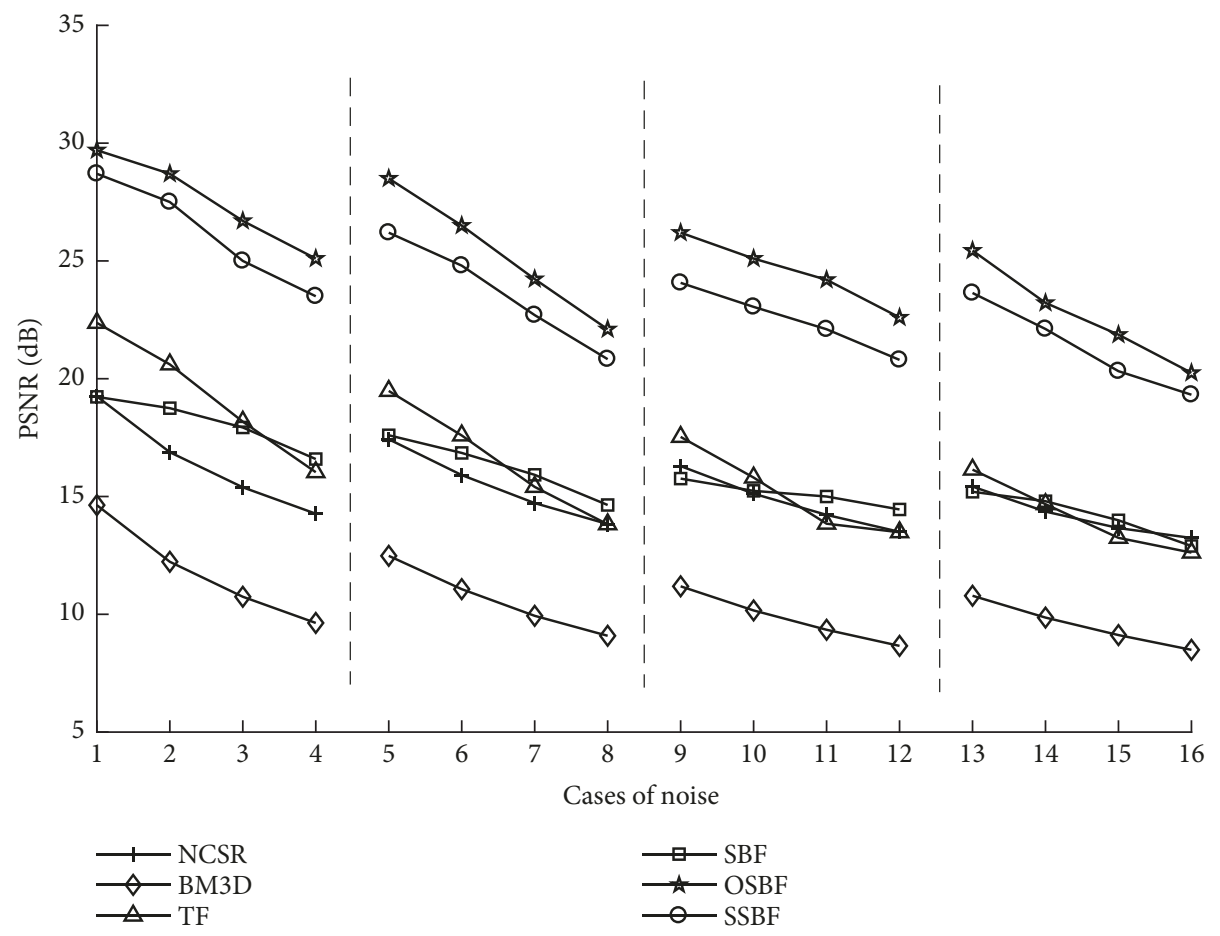

FIGURE 13: PSNR comparison of NCSR, BM3D, TF, SBF, OSBF, and SSBF for image that is corrupted speckle noise mixed with salt-and-pepper noise.

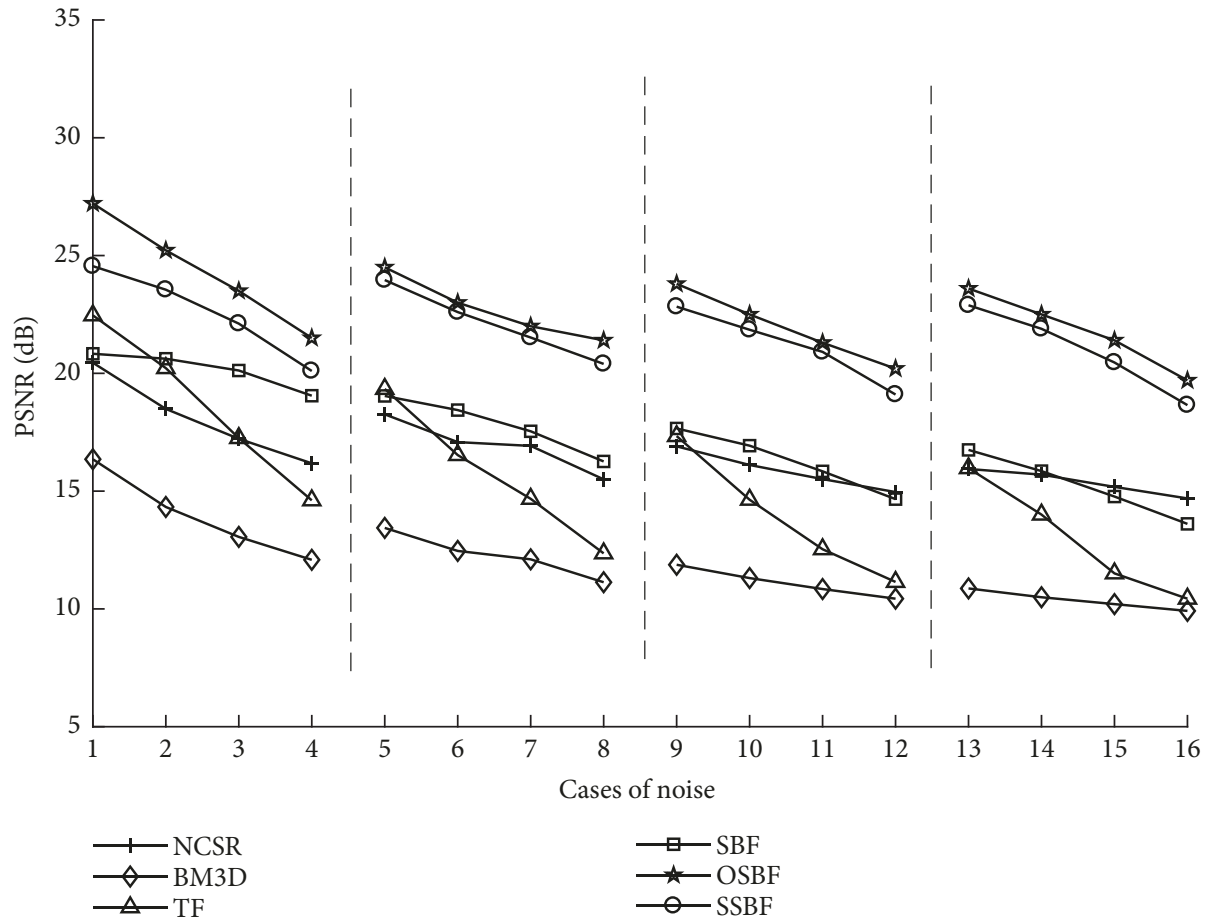

FIGURE 14: PSNR comparison of NCSR, BM3D, TF, SBF, OSBF, and SSBF for image that is corrupted speckle noise mixed with uniform impulse noise.

for removing such kind of speckle noise. Therefore, they give poor performance.

As a result, NCSR, TF, and SBF filters work well on reasonable noise strength. When noise strength becomes higher, their performance significantly drops. This is because they cannot distinguish noise and true signal effectively.
Moreover, the important function of mixed noise filter is noise identification; when the strength of noise is high, NCSR, TF, and SBF filters cannot identify noise type accurately. Therefore, their performance suffers from this inaccurate noise identification. Moreover, NCSR, BM3D, TF, and SBF filters did not consider the speckle and salt-and-pepper 


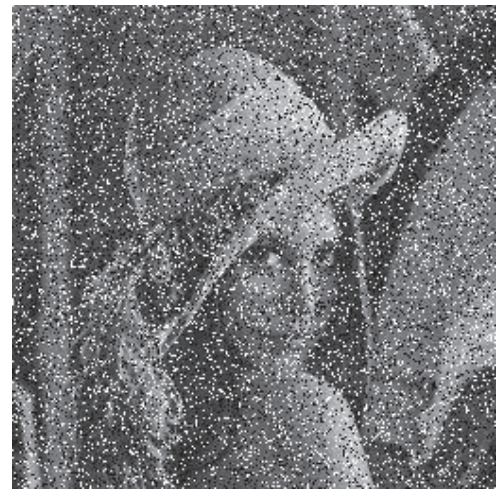

(a)

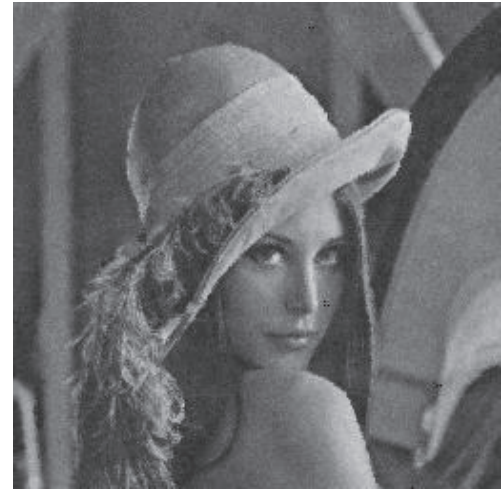

(b)

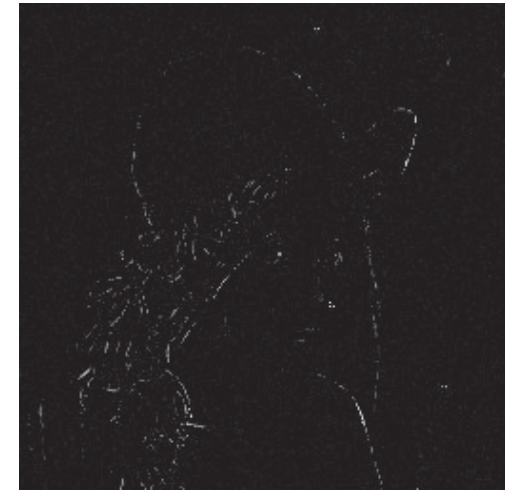

(c)

FIGURE 15: A performance of SSBF of Lena image: (a) Group A: noisy image by Gaussian noise (SNR = $25 \mathrm{~dB})$ mixed with salt-and-pepper noise $(\rho=20 \%)$, (b) output from filter (PSNR $=29.34 \mathrm{~dB}$ and SSIM $=0.8495)$, and (c) the distinction between (a) and (b).

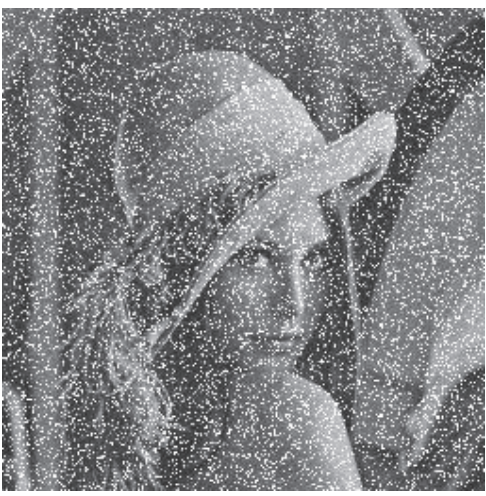

(a)

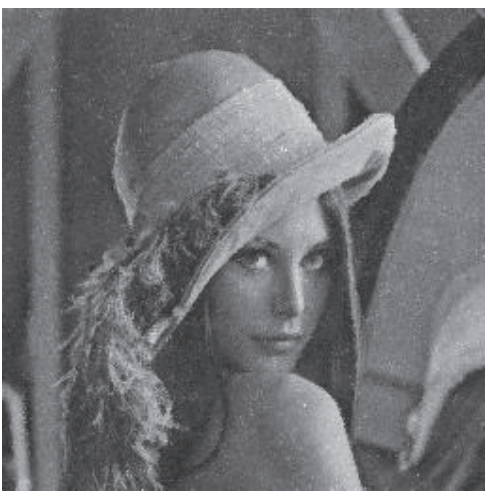

(b)

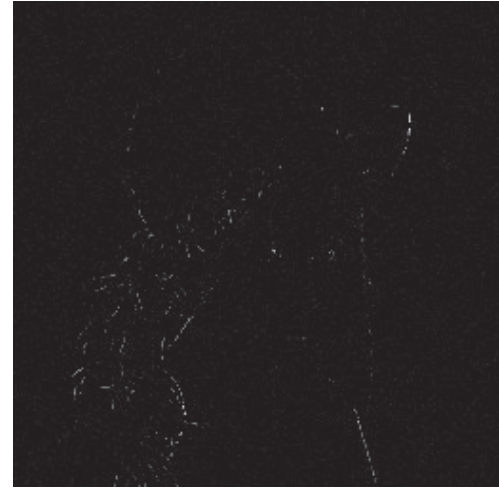

(c)

FIGURE 16: A performance of SSBF of Lena image: (a) Group B: noisy image by Gaussian noise (SNR = $25 \mathrm{~dB}$ ) mixed with uniform impulse noise $(\rho=20 \%)$, (b) output from filter (PSNR $=28.81 \mathrm{~dB}$ and SSIM $=0.7536$ ), and (c) the distinction between (a) and (b).

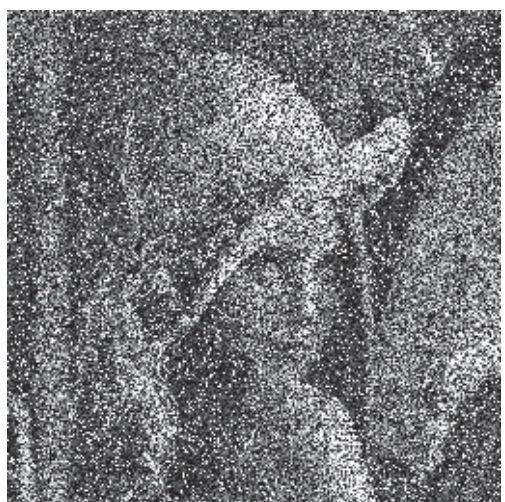

(a)

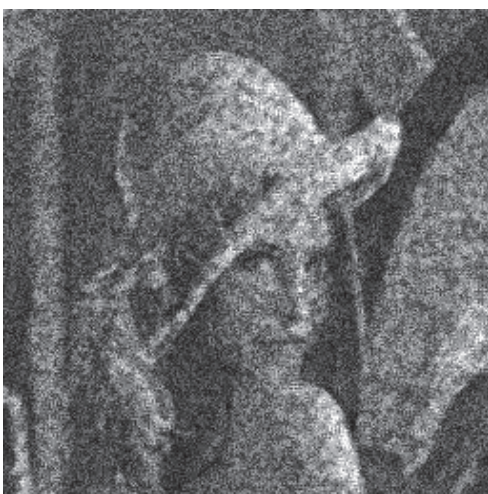

(b)

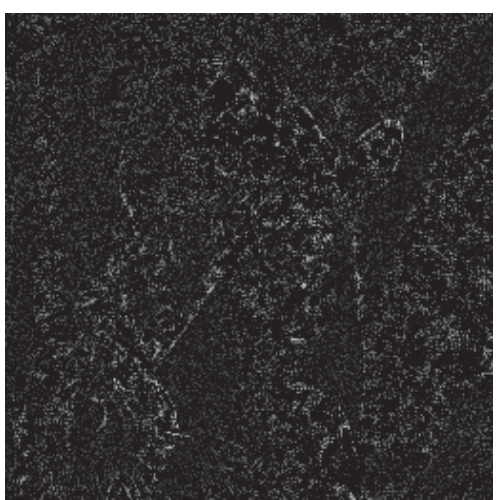

(c)

FIGURE 17: A performance of SSBF of Lena image: (a) Group C: noisy image by speckle noise ( $\rho=20 \%)$ mixed with salt-and-pepper noise ( $\rho$ $=20 \%)$, (b) output from filter (PSNR $=25.40 \mathrm{~dB}$ and SSIM = 0.6703), and (c) the distinction between (a) and (b).

noise; then they give poor performance when the image is contaminated with speckle and salt-and-pepper noise. The simulation results show that our proposed filter (SSBF) gives the highest PSNR for all groups of mixed noise since it takes the four types of noise into account in order to perform noise estimation and to remove.
To illustrate the output from the SSBF, two learned images-Lena and Pentagon-and a nonlearning imagePepper-are contaminated with the four groups of mixed noise and performed noise removal as shown in Figures 15-26. For convenience on noticing the performance, the absolute differences between original and output images 


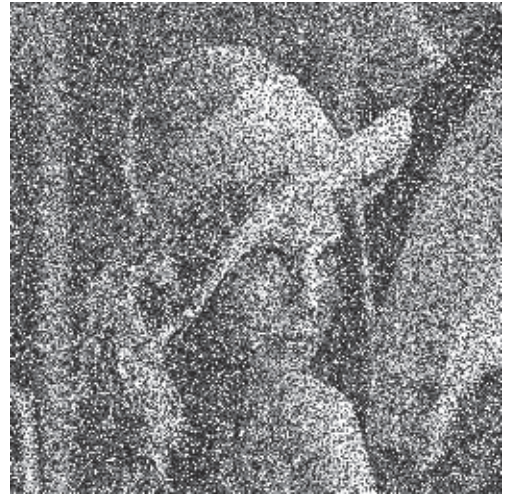

(a)

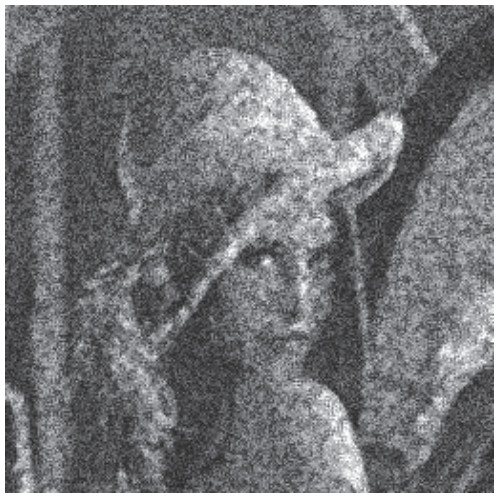

(b)

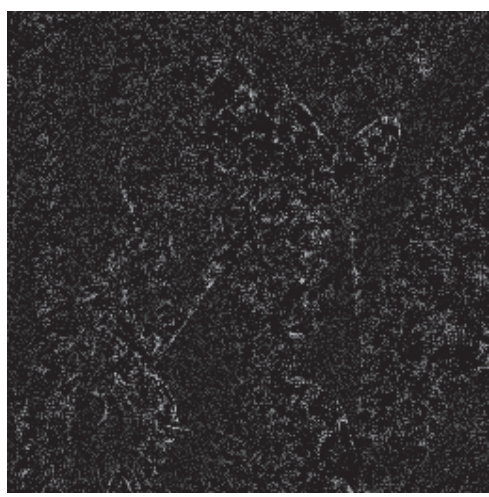

(c)

FIGURE 18: A performance of SSBF of Lena image: (a) Group D: noisy image by speckle noise $(\rho=20 \%)$ mixed with uniform impulse noise $(\rho=20 \%)$, (b) output from filter (PSNR $=23.03 \mathrm{~dB}$ and SSIM $=0.5705)$, and (c) the distinction between (a) and (b).

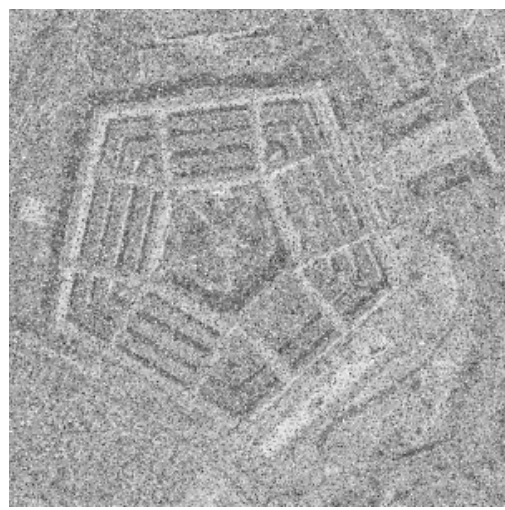

(a)

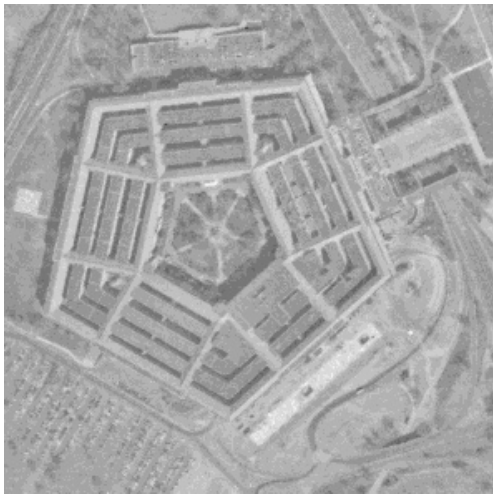

(b)

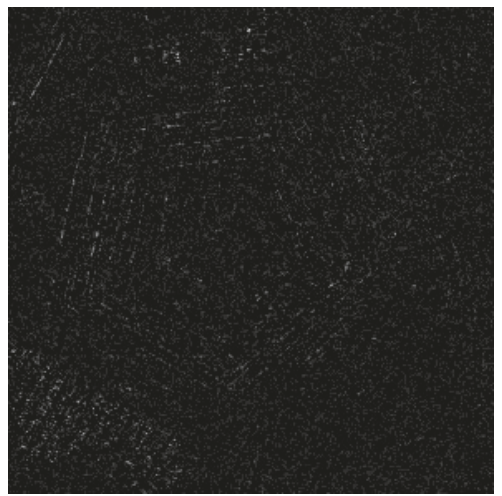

(c)

FIGURE 19: A performance of SSBF of Pentagon image: (a) Group A: noisy image by Gaussian noise (SNR $=25 \mathrm{~dB}$ ) mixed with salt-and-pepper noise $(\rho=20 \%)$, (b) output from filter (PSNR $=28.22 \mathrm{~dB}$ and SSIM $=0.7506)$, and (c) the distinction between (a) and (b).

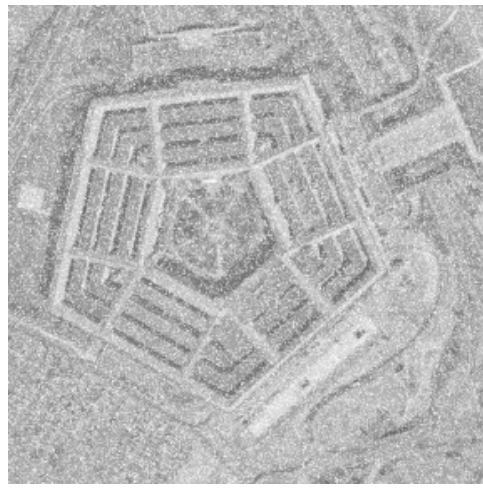

(a)

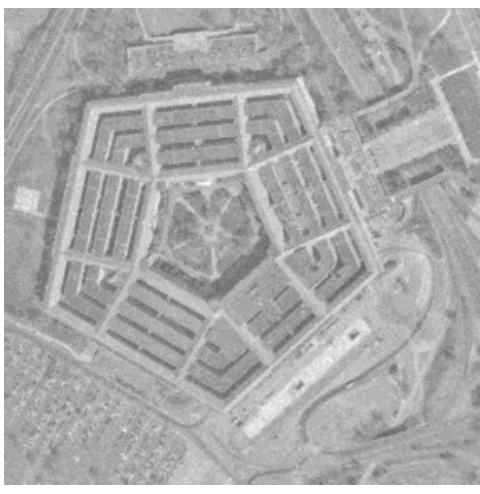

(b)

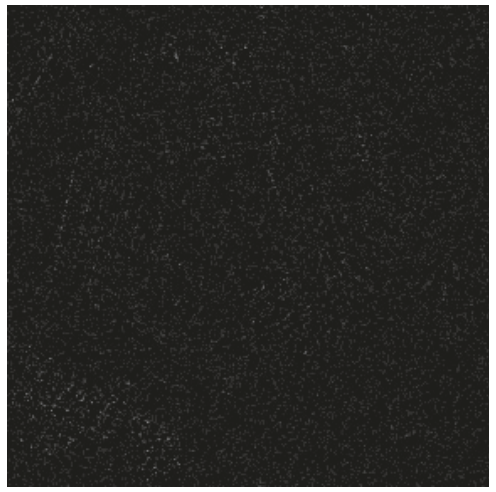

(c)

Figure 20: A performance of SSBF of Pentagon image: (a) Group B: noisy image by Gaussian noise (SNR $=25 \mathrm{~dB}$ ) mixed with uniform impulse noise ( $\rho=20 \%)$, (b) output from filter (PSNR $=26.13 \mathrm{~dB}$ and SSIM $=0.6983$ ), and (c) the distinction between (a) and (b).

are plotted in subfigure (c) for all figures. As a result, once the type and strength of mixed noise are estimated efficiently, SSBF can perform noise removal with the appropriate weights, spatial weight $\left(\sigma_{\text {S.est }}\right)$ and radiometric weight $\left(\sigma_{\text {R.est }}\right)$, for the contaminated mixed noise. As depicted by the distinction between the original image and filtered image, it can be seen that SSBF removes mixed noise while persevering edges and details of the image. To illustrate the performance 


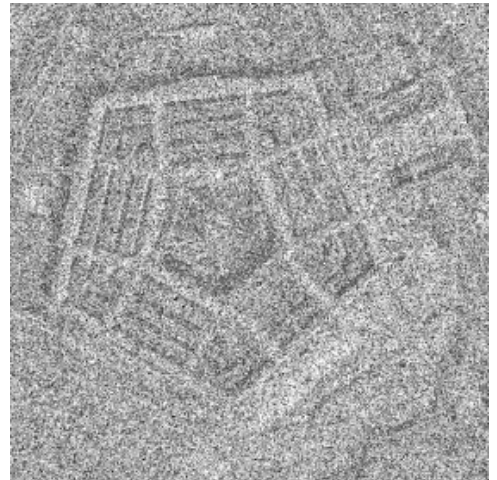

(a)

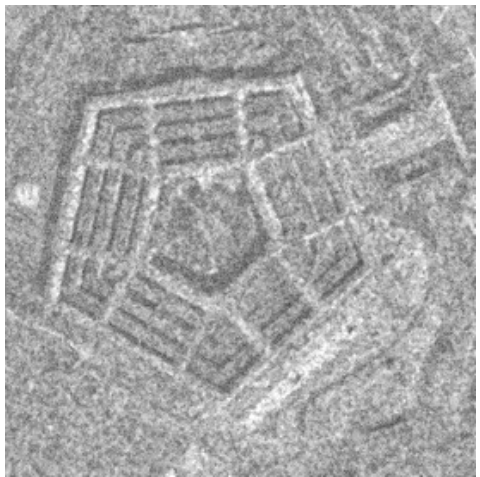

(b)

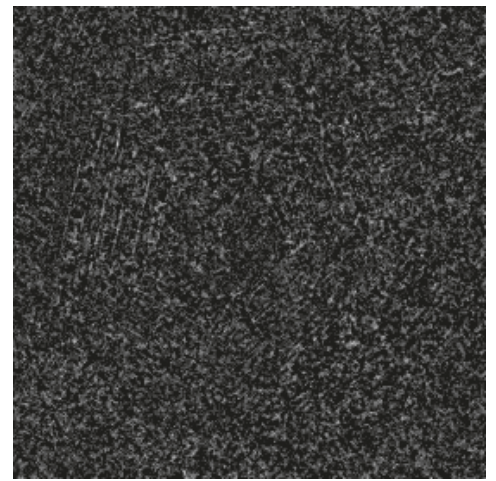

(c)

Figure 21: A performance of SSBF of Pentagon image: (a) Group C: noisy image by speckle noise $(\rho=20 \%)$ mixed with salt-and-pepper noise $(\rho=20 \%)$, (b) output from filter (PSNR $=24.56 \mathrm{~dB}$ and SSIM $=0.6249)$, and (c) the distinction between (a) and (b).

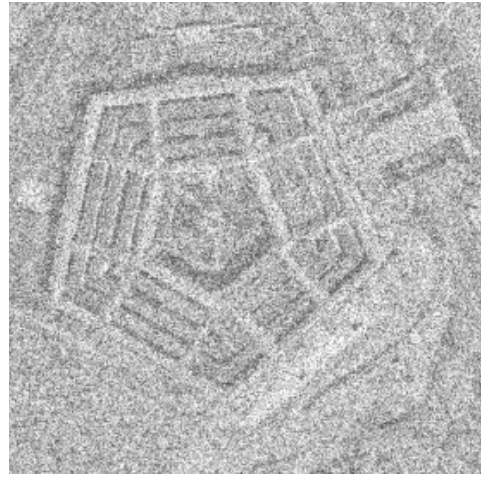

(a)

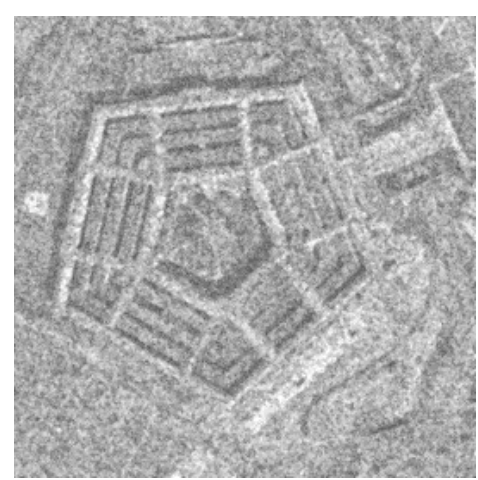

(b)

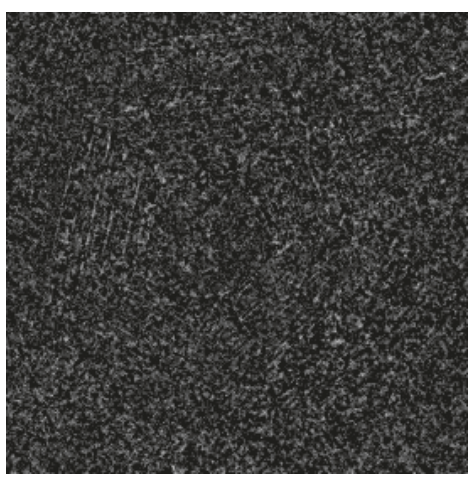

(c)

FIGURE 22: A performance of SSBF of Pentagon image: (a) Group D: noisy image by speckle noise $(\rho=20 \%)$ mixed with uniform impulse noise $(\rho=20 \%)$, (b) output from filter (PSNR $=22.53 \mathrm{~dB}$ and SSIM $=0.5768$ ), and (c) the distinction between (a) and (b).

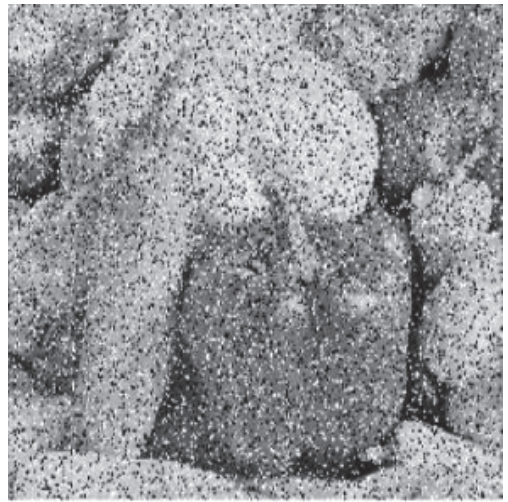

(a)

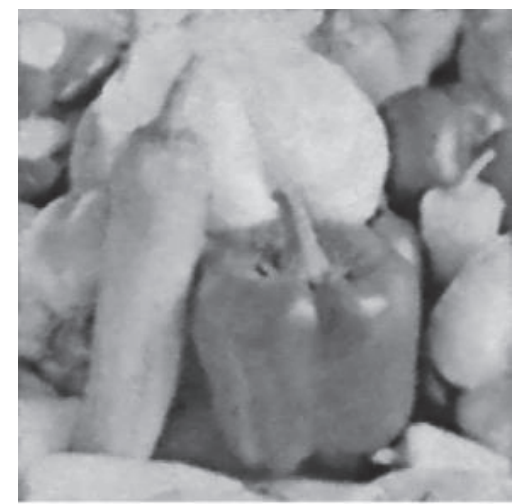

(b)

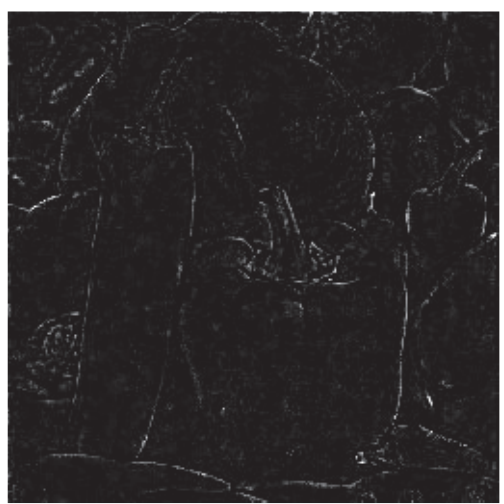

(c)

FIGURE 23: A performance of SSBF of Pepper image: (a) Group A: noisy image by Gaussian noise (SNR $=25 \mathrm{~dB})$ mixed with salt-and-pepper noise $(\rho=20 \%)$, (b) output from filter (PSNR $=28.72 \mathrm{~dB}$ and SSIM=0.7495), and (c) the distinction between (a) and (b).

of these filters, the outputs from NCSR, BM3D, TF, SBF, OSBF, and SSBF in a zoomed version of the learned image, Lena, and nonlearning image, Pepper, are compared in Figures 27 and 28 , respectively. The simulation results show that SSBF can remove the mixed noise while preserving edge effectively.

\section{Conclusion}

In this paper, we propose a smart switching bilateral filter (SSBF) and an estimated noise characterization scheme for mixed noise removal. For noise estimation, the estimated 


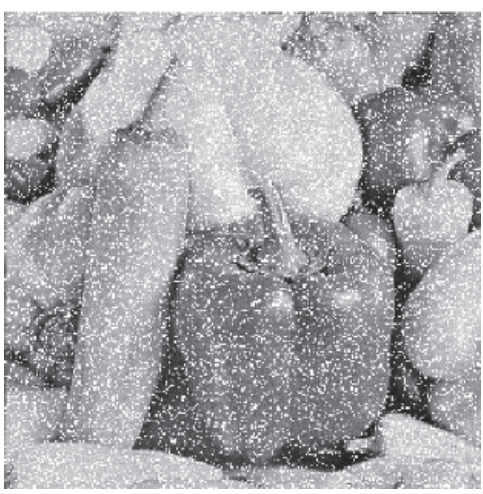

(a)

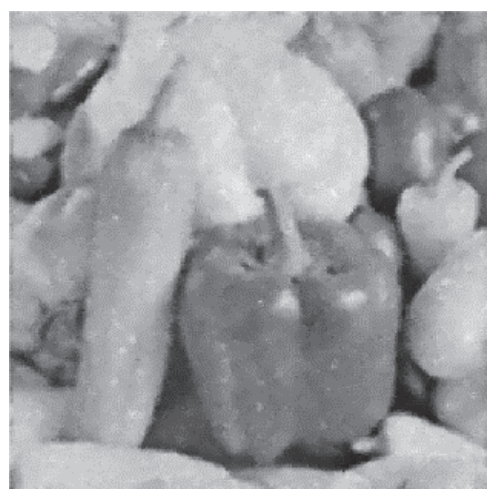

(b)

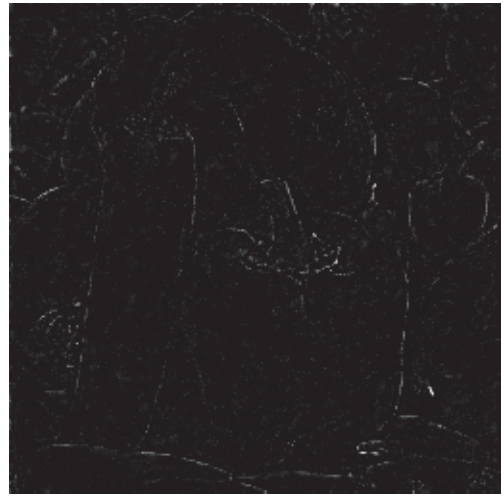

(c)

FIgURE 24: A performance of SSBF of Pepper image: (a) Group B: noisy image by Gaussian noise (SNR $=25 \mathrm{~dB}$ ) mixed with uniform impulse noise $(\rho=20 \%)$, (b) output from filter (PSNR $=28.15 \mathrm{~dB}$ and SSIM $=0.7172)$, and (c) the distinction between (a) and (b).

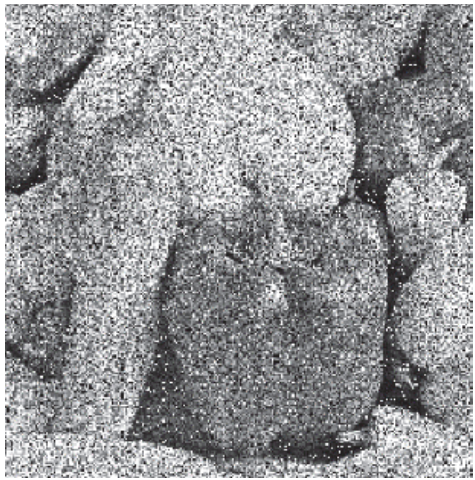

(a)

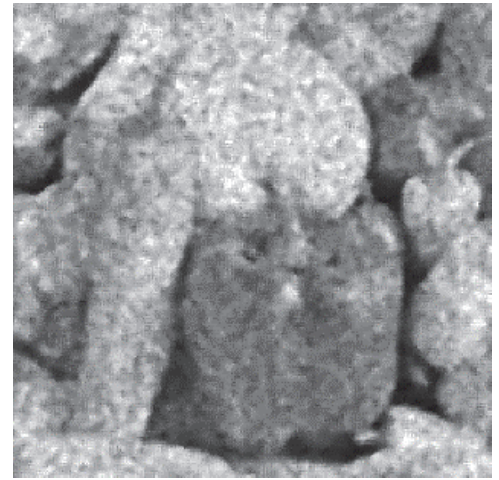

(b)

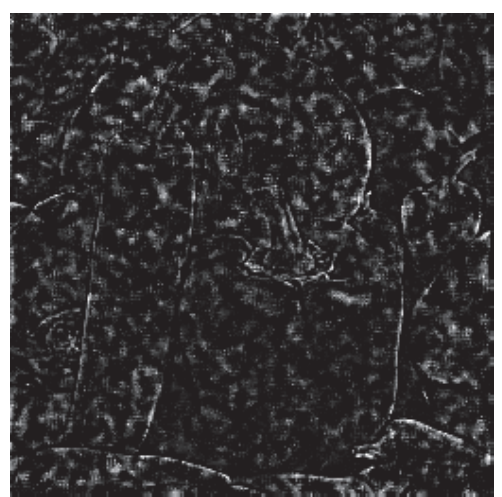

(c)

FIgURE 25: A performance of SSBF of Pepper image: (a) Group C: noisy image by speckle noise $(\rho=20 \%)$ mixed with salt-and-pepper noise $(\rho=20 \%)$, (b) output from filter (PSNR $=22.14 \mathrm{~dB}$ and SSIM $=0.5650)$, and (c) the distinction between (a) and (b).

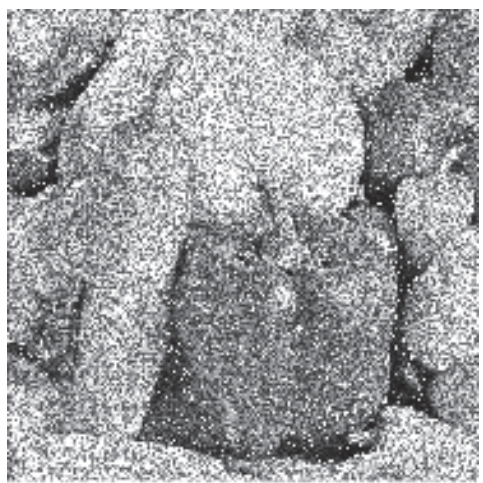

(a)

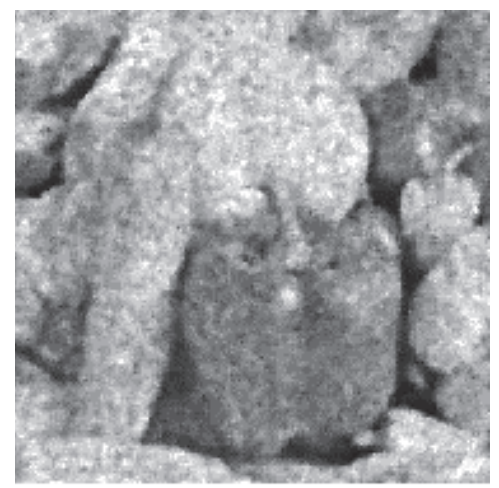

(b)

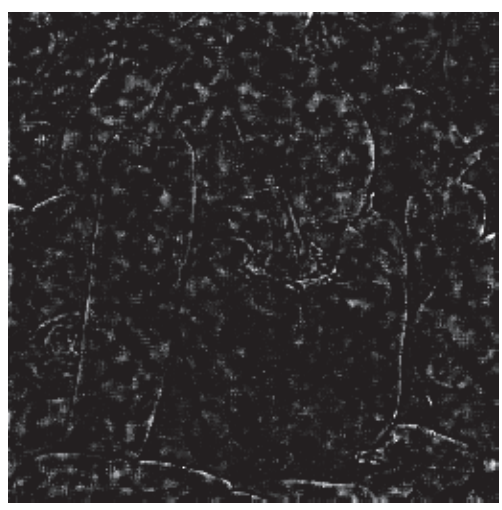

(c)

FIGURE 26: A performance of SSBF of Pepper image: (a) Group D: noisy image by speckle noise $(\rho=20 \%)$ mixed with uniform impulse noise $(\rho=20 \%)$, (b) output from filter (PSNR $=22.39 \mathrm{~dB}$ and SSIM $=0.5732)$, and (c) the distinction between (a) and (b).

noise characterization scheme analyzes the mixed noise from the distribution of the difference between center pixel and neighbors; it gives an accurate estimation greater than $80 \%$. For a SSBF, once the mixed noise type and strength are estimated accurately, the filter parameters are selected from the parameters table where the parameters are already learned for the detected mixed noise. Therefore, the SSBF gives high noise removal performance without knowing the type and strength of mixed noise. By comparing SSBF to nonlocal sparse representation (NCSR), block-matching and 


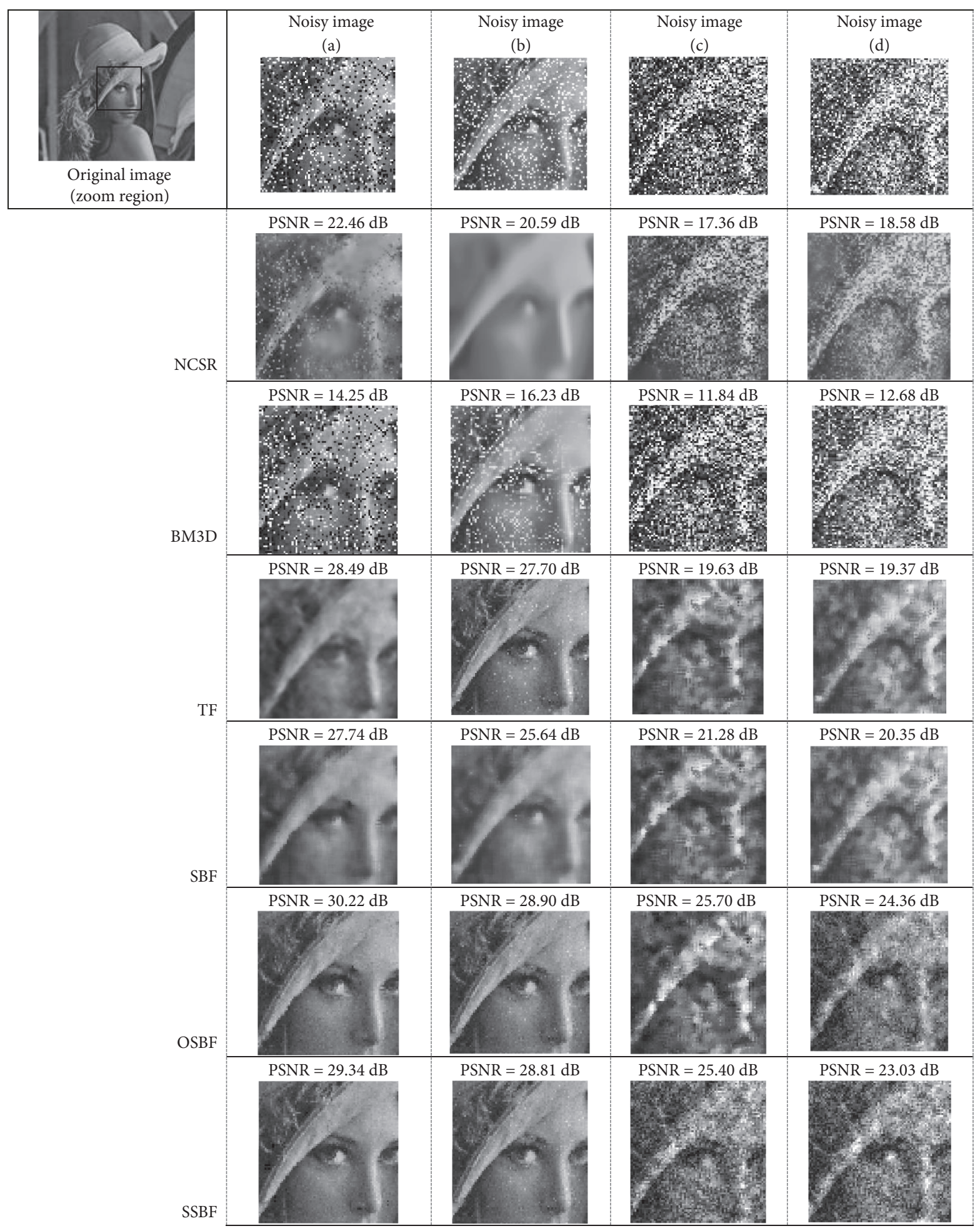

FIGURE 27: Compare PSNR of NCSR, BM3D, TF, SBF, OSBF, and SSBF for Lena image. (a) Group A: noisy image by Gaussian noise (SNR = $25 \mathrm{~dB}$ ) mixed with salt-and-pepper noise $(\rho=20 \%)$, (b) Group B: noisy image by Gaussian noise (SNR $=25 \mathrm{~dB}$ ) mixed with uniform impulse noise $(\rho=20 \%)$, (c) Group C: noisy image by speckle noise $(\rho=20 \%)$ mixed with salt-and-pepper noise $(\rho=20 \%)$, and (d) Group D: noisy image by speckle noise $(\rho=20 \%)$ mixed with uniform impulse noise $(\rho=20 \%)$. 


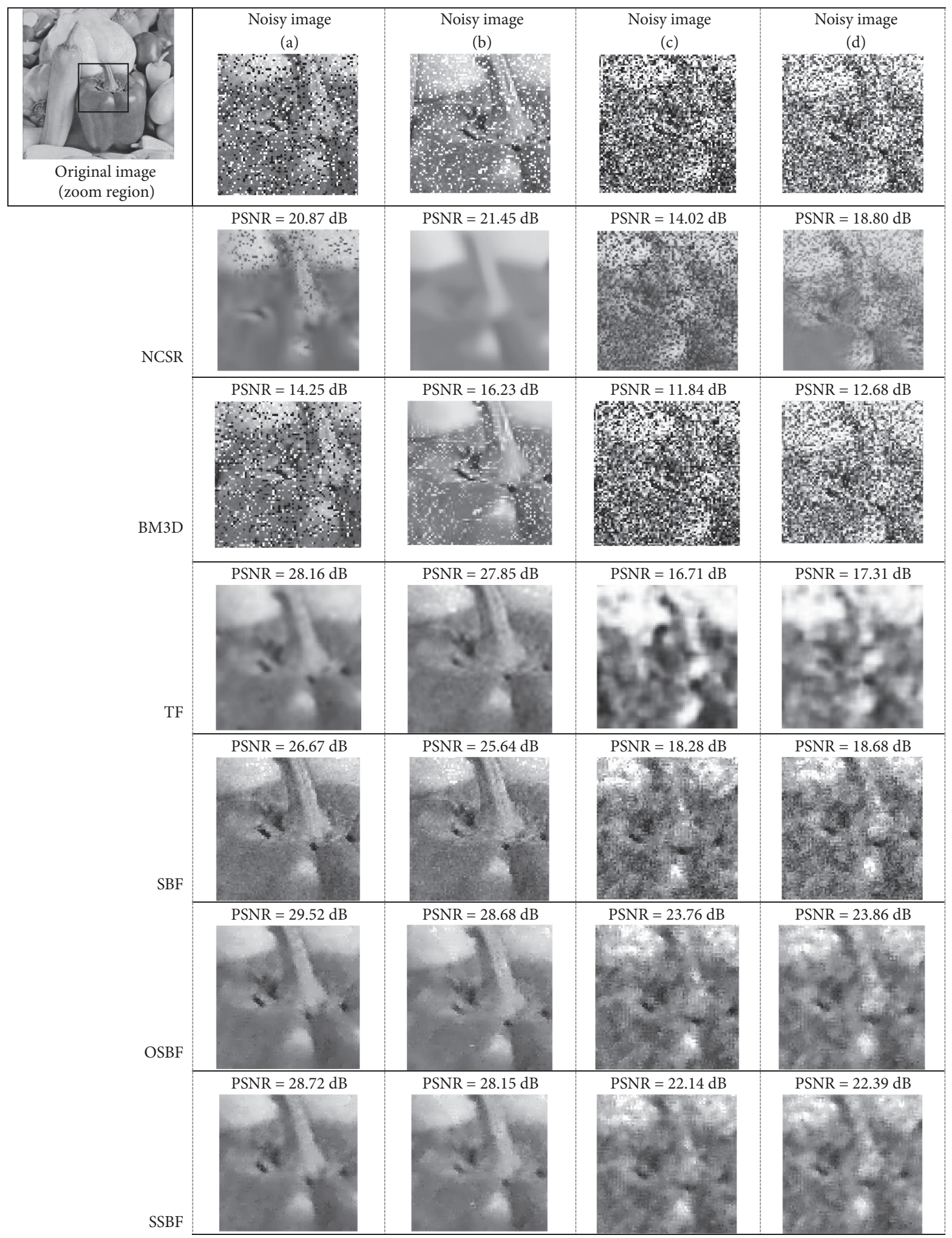

FIGURE 28: Compare PSNR of NCSR, BM3D, TF, SBF, OSBF, and SSBF for Pepper image; (a) Group A: noisy image by Gaussian noise (SNR = $25 \mathrm{~dB}$ ) mixed with salt-and-pepper noise $(\rho=20 \%)$, (b) Group B: noisy image by Gaussian noise (SNR $=25 \mathrm{~dB})$ mixed with uniform impulse noise $(\rho=20 \%)$, (c) Group C: noisy image by speckle noise $(\rho=20 \%)$ mixed with salt-and-pepper noise $(\rho=20 \%)$, and (d) Group D: noisy image by speckle noise $(\rho=20 \%)$ mixed with uniform impulse noise $(\rho=20 \%)$. 
3D filtering (BM3D), trilateral filter (TF), and switching bilateral filter $(\mathrm{SBF})$, it can be seen that once the noise type is identified accurately and the filter weights are selected appropriately, SSBF improves a noise removal performance of TF and SBF filter and also outperforms all compared techniques.

\section{Data Availability}

No data were used to support this study.

\section{Conflicts of Interest}

There are no conflicts of interest regarding the publication of this paper.

\section{Acknowledgments}

This work was partially supported by Ministry of Science and Technology of Thailand.

\section{References}

[1] K. Jain, Fundamentals of Digital Image Processing, Information and System Sciences Series, Prentice-Hall, Upper Saddle River, NJ, USA, 1989.

[2] R. C. Gonzalez and R. E. Woods, Digital Image Processing, Pearson Prentice Hall, NJ, USA, 3rd edition, 2008.

[3] W. K. Pratt, Digital Image Processing, John Wiley \& Sons, Inc., New York, NY, USA, 1978.

[4] L. Shao, R. Yan, X. Li, and Y. Liu, "From heuristic optimization to dictionary learning: a review and comprehesive comparison of image denoising algorithms," IEEE Transactions on Image Processing, pp. 1001-1013, 2014.

[5] J.-G. Camarena, V. Gregori, S. Morillas, and A. Sapena, "A simple fuzzy method to remove mixed Gaussian-impulsive noise from colour images," IEEE Transactions on Fuzzy Systems, vol. 21, no. 5, pp. 971-978, 2013.

[6] S. Morillas, V. Gregori, and A. Hervás, "Fuzzy peer groups for reducing mixed Gaussian-impulse noise from color images," IEEE Transactions on Image Processing, vol. 18, no. 7, pp. 14521466, 2009.

[7] L. Hou, H. Liu, Z. Luo, Y. Zhou, and T. Truong, "Image deblurring in the presence of salt-and-pepper noise," IEEE Transactions on Image Processing, pp. 2389-2393, 2017.

[8] T. Yassine, S. Ahmed, and N. Abdelkrim, "Speckle noise reduction in digital speckle pattern interferometry using Riesz wavelets transform," in Proceedings of the $3 \mathrm{rd}$ International Conference on Advanced Technologies for Signal and Image Processing, ATSIP 2017, pp. 1-4, Morocco, May 2017.

[9] J. Jiang, L. Zhang, and J. Yang, "Mixed noise removal by weighted encoding with sparse nonlocal regularization," IEEE Transactions on Image Processing, vol. 23, no. 6, pp. 2651-2662, 2014.

[10] I. R. Terol-Villalobos and J. D. Mendiola-Santibanez, "Filtering of mixed Gaussian and impulsive noise using morphological contrast detectors," IET Image Processing, pp. 131-141, 2014.

[11] S. Ramesh., "An efficient approach for removal of universal noise using adaptive based switching bilateral filter," in Proceedings of the 1st International Conference on Advances in
Engineering, Science and Management, ICAESM-2012, pp. 462467, India, March 2012.

[12] Y. Zhou, Z. Ye, and Y. Xiao, "A restoration algorithm for images contminated by mixed Gaussian plus random-values impulse noise," Journal of Visual Communication and Image Representation, pp. 283-294, 2013.

[13] J.-G. Camarena, V. Gregori, S. Morillas, and A. Sapena, "A simple fuzzy method to remove mixed Gaussian-impulsive noise from color images," IEEE Transactions on Fuzzy Systems, vol. 21, no. 5, pp. 971-978, 2013.

[14] B. K. Shreyamsha-Kumar, "Image denoising based on gaussian/bilateral filter and its method noise thresholding," Signal, Image and Video Processing, vol. 7, no. 6, pp. 1159-1172, 2013.

[15] C. Xiong, L. Chen, and Y. Pang, "An adaptive bilateral filtering algorithm and its application in edge detection," in Proceedings of the International Conference on Measuring Technology and Mechatronics Automation, ICMTMA 2010, pp. 440-443, China, March 2010.

[16] L. Caraffa, J.-P. Tarel, and P. Charbonnier, "The guided bilateral filter: when the joint/cross bilateral filter becomes robust," IEEE Transactions on Image Processing, vol. 24, no. 4, pp. 1199-1208, 2015.

[17] P. D. Patil and A. D. Kumbhar, "Bilateral filter for image denoising," in Proceedings of the 1st International Conference on Green Computing and Internet of Things, ICGCIoT 2015, pp. 299-302, India, October 2015.

[18] L. Ying-hui, G. Kun, and N. Guo-qiang, "An improved trilateral filter for Gaussian and impulse noise removal," in Proceedings of the 2010 2nd International Conference on Industrial Mechatronics and Automation, ICIMA 2010, vol. 2, pp. 385-388, China, May 2010.

[19] H.-H. Chang, "Entropy-based trilateral filtering for noise removal in digital images," in Proceedings of the $20103 \mathrm{rd}$ International Congress on Image and Signal Processing, CISP 2010, pp. 673-677, China, October 2010.

[20] T. Azetsu, N. Suetake, and E. Uchino, “Trilateral filter using rank order information of pixel value for mixed Gaussian and impulsive noise removal," in Proceedings of the 2013 21st International Symposium on Intelligent Signal Processing and Communication Systems, ISPACS 2013, pp. 303-306, Japan, November 2013.

[21] V. Patanavijit and K. Thakulsukanant, "Dissection capability analysis of noise suppression technique utilizing trilateral filter under mixed noise circumstances," in Proceedings of the 14th International Conference on Electrical Engineering/Electronics, Computer, Telecommunications and Information Technology, ECTI-CON 2017, pp. 119-122, Thailand, June 2017.

[22] C.-H. Lin, J.-S. Tsai, and C.-T. Chiu, "Switching bilateral filter with a texture/noise detector for universal noise removal," IEEE Transactions on Image Processing, vol. 19, no. 9, pp. 2307-2320, 2010.

[23] K. S. Rani and R. V. S. Satyanarayana, "Image denoising using boundary discriminated switching bilateral filter with highly corrupted universal noise," in Proceedings of the 2017 International Conference on Energy, Communication, Data Analytics and Soft Computing, ICECDS 2017, pp. 1515-1521, India, August 2017.

[24] V. Jayaraj, D. Ebenezer, and N. K. Prakash, "A switching bilateral filter for images with edge preservation properties," in Proceedings of the 2010 2nd International Conference on Signal Processing Systems (ICSPS), vol. 2, pp. 435-439, Dalian, China, July 2010. 
[25] K. Langampol, W. Lee, and V. Patanavijit, "The switching bilateral denoising performance influence of spatial and radiometric variance," in Proceedings of the 13th International Conference on Electrical Engineering/Electronics, Computer, Telecommunications and Information Technology, ECTI-CON 2016, pp. 1-6, Thailand, July 2016.

[26] P. Jain and V. Tyagi, "LAPB: locally adaptive patch-based wavelet domain edge-preserving image denoising," Information Sciences, vol. 294, pp. 164-181, 2015.

[27] M. Maggioni, G. Boracchi, A. Foi, and K. Egiazarian, "Video denoising, deblocking, and enhancement through separable 4D nonlocal spatiotemporal transforms," IEEE Transactions on Image Processing, vol. 21, no. 9, pp. 3952-3966, 2012.

[28] K. Dabov, A. Foi, V. Katkovnik, and K. Egiazarian, "BM3D image denoising with shape-adaptive principal component analysis," in Proceedings of the Signal Processing with Adaptive Sparse Structured Representations (SPARS'09), pp. 1-6, 2009.

[29] W. Dong, L. Zhang, G. Shi, and X. Li, "Nonlocally centralized sparse representation for image restoration," IEEE Transactions on Image Processing, vol. 22, no. 4, pp. 1620-1630, 2013.

[30] A. Palacios, V. Ponomaryov, R. Reyes-Reyes, and S. Sadovnychiy, "Sparse technique for images corrupted by mixed Gaussian-impulsive noise," Circuits, Systems and Signal Processing, vol. 37, no. 12, pp. 5389-5416, 2018.

[31] V. F. Kravchenko, V. I. Ponomaryov, V. I. Pustovoit, and A. Palacios-Enriquez, "Complex noise suppression using a sparse representation and 3D filtering of images," Doklady Physics, vol. 62, no. 8, pp. 379-383, 2017.

[32] V. F. Kravchenko, V. I. Ponomaryov, and V. I. Pustovoit, "3D filtering of images corrupted by mixed additive-impulsive noise," Doklady Physics, vol. 63, no. 8, pp. 321-325, 2018.

[33] A. Horé and D. Ziou, "Image quality metrics: PSNR vs. SSIM," in Proceedings of the 20th International Conference on Pattern Recognition (ICPR '10), pp. 2366-2369, Istanbul, Turkey, August 2010.

[34] Y. Zhang, X. Tian, and P. Ren, "An adaptive bilateral filter based framework for image denoising," Neurocomputing, vol. 140, pp. 299-316, 2014.

[35] C. Chang, J. Hsiao, and C. Hsieh, "An adaptive median filter for image denoising," in Proceedings of the 2nd International Symposium on Intelligent Information Technology Application (IITA '08), vol. 2, pp. 346-350, Shanghai, China, December 2008.

[36] G. George, R. M. Oommen, S. Shelly, S. S. Philipose, and A. M. Varghese, "A survey on various median filtering techniques for removal of impulse noise from digital image," in Proceedings of the 2018 Conference on Emerging Devices and Smart Systems (ICEDSS), pp. 235-238, Tiruchengode, India, March 2018. 


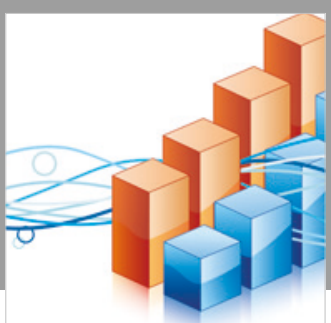

Advances in

Operations Research

\section{-n-m}
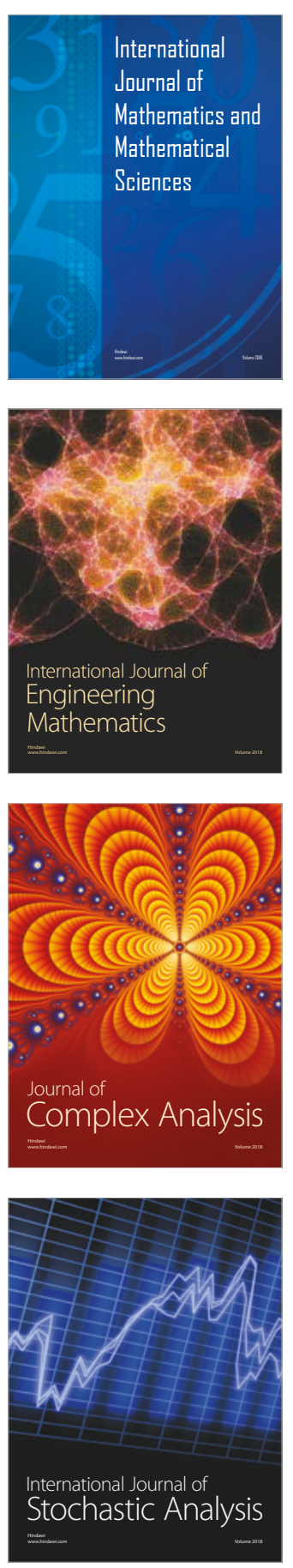
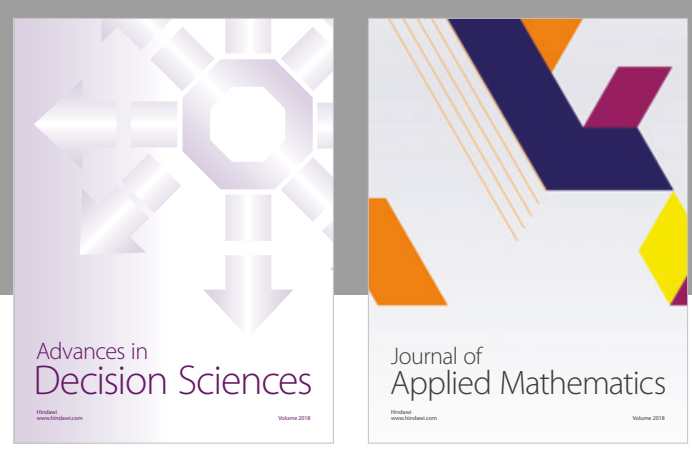

Journal of

Applied Mathematics
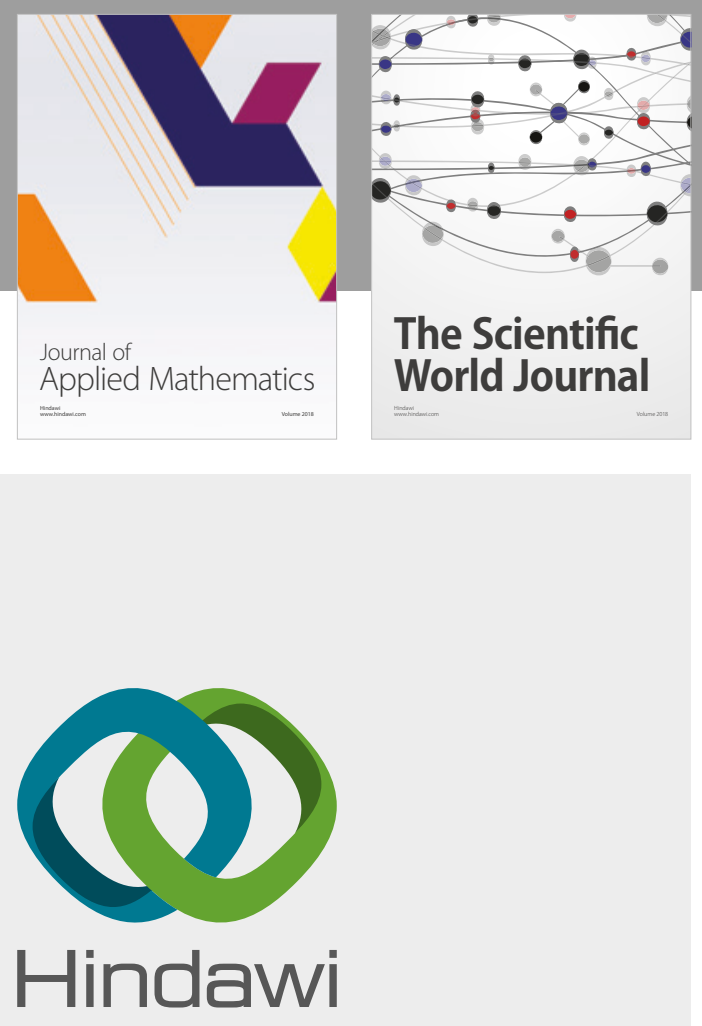

Submit your manuscripts at

www.hindawi.com

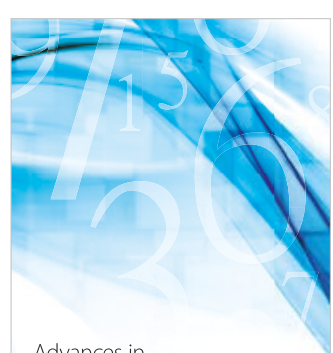

Advances in
Numerical Analysis
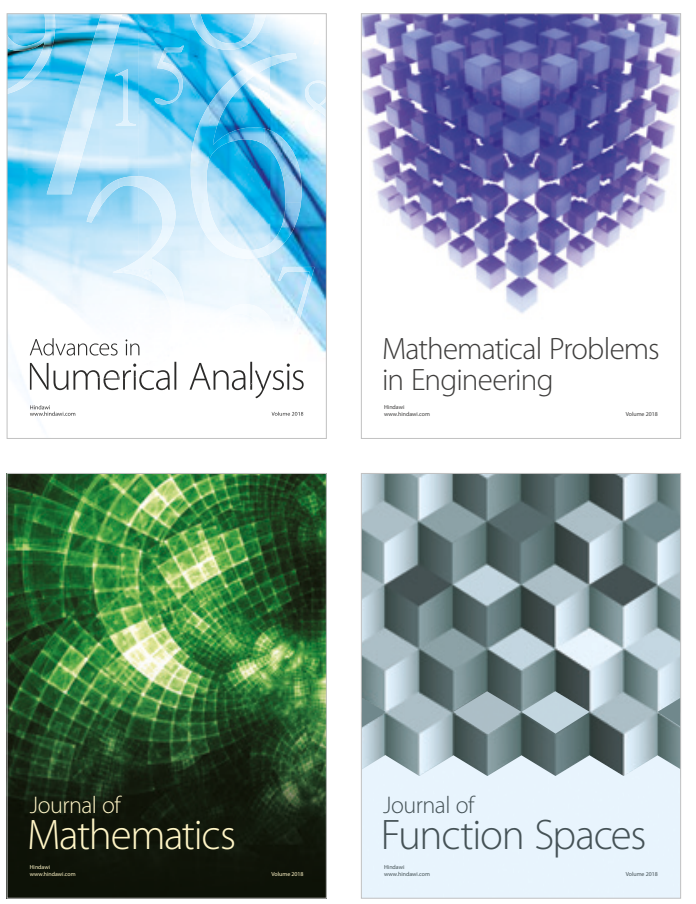

Mathematical Problems in Engineering

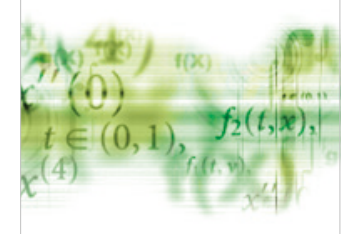

International Journal of

Differential Equations

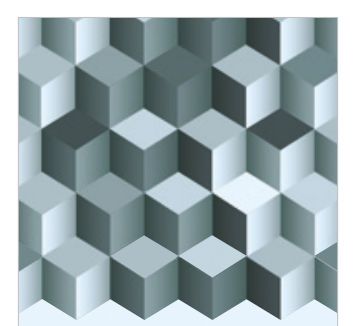

Journal of

Function Spaces

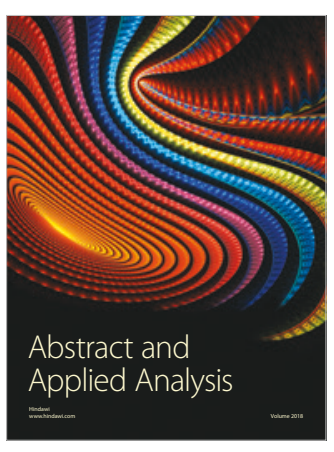

The Scientific

World Journal

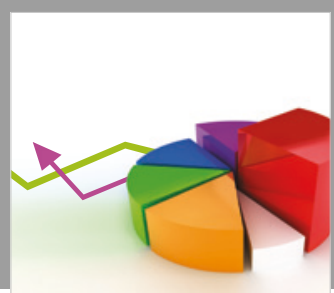

Journal of

Probability and Statistics
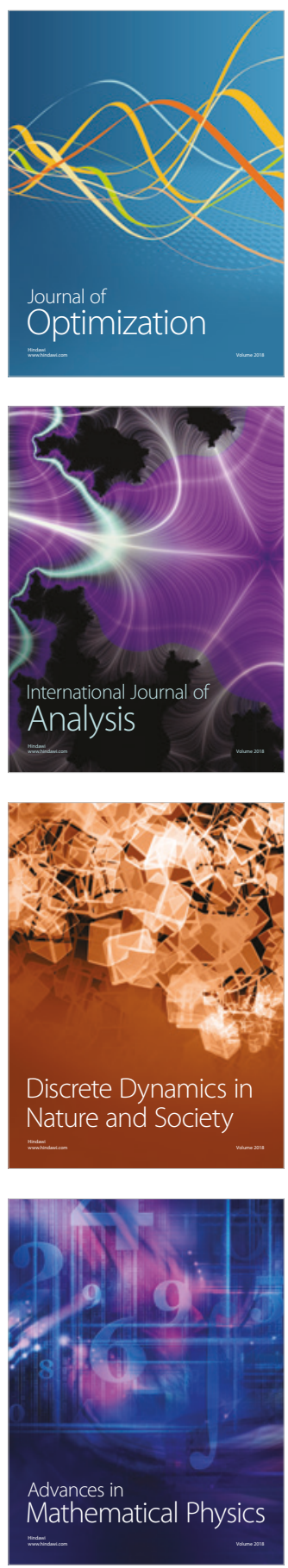